\title{
REPRESENTATIONS OF THE KAUFFMAN BRACKET SKEIN ALGEBRA II: PUNCTURED SURFACES
}

\author{
FRANCIS BONAHON AND HELEN WONG
}

\begin{abstract}
In earlier work $\mathrm{BoW}_{3}$, we constructed invariants of irreducible finite-dimensional representations of the Kauffman bracket skein algebra of a surface. We introduce here an inverse construction, which to a set of possible invariants associates an irreducible representation that realizes these invariants. The current article is restricted to surfaces with at least one puncture, a condition that is lifted in subsequent work $\mathrm{BoW}_{6}$ relying on this one. A step in the proof is of independent interest, and describes the arithmetic structure of the Thurston intersection form on the space of integer weight systems for a train track.
\end{abstract}

This article is a continuation of $\left[\mathrm{BoW}_{3}\right]$ and is part of the program described in $\left[\mathrm{BoW}_{2}\right.$, devoted to the analysis and construction of finite-dimensional representations of the Kauffman bracket skein algebra of a surface.

Let $S$ be an oriented surface of finite topological type without boundary. The Kauffman bracket skein algebra $\mathcal{S}^{A}(S)$ depends on a parameter $A=\mathrm{e}^{\mathrm{\pi i} \hbar} \in \mathbb{C}-\{0\}$, and is defined as follows: One first considers the vector space freely generated by all isotopy classes of framed links in the thickened surface $S \times[0,1]$, and then one takes the quotient of this space by two relations. The first and main relation is the skein relation, which states that

$$
\left[K_{1}\right]=A^{-1}\left[K_{0}\right]+A\left[K_{\infty}\right]
$$

whenever the three links $K_{1}, K_{0}$ and $K_{\infty} \subset S \times[0,1]$ differ only in a little ball where they are as represented in Figure 1. The second relation is the trivial knot relation, which asserts that

$$
[K \cup O]=-\left(A^{2}+A^{-2}\right)[K]
$$

whenever $O$ is the boundary of a disk $D \subset K \times[0,1]$ disjoint from $K$, and is endowed with a framing transverse to $D$. The algebra multiplication is provided by the operation of superposition, for which the product $[K] \cdot[L]$ is represented by the union $\left[K^{\prime} \cup L^{\prime}\right]$ where $K^{\prime} \subset S \times\left[0, \frac{1}{2}\right]$ and $L^{\prime} \subset S \times\left[\frac{1}{2}, 1\right]$ are respectively obtained by rescaling the framed links $K \subset S \times[0,1]$ and $L^{\prime} \subset S \times[0,1]$ in the $[0,1]$ direction.

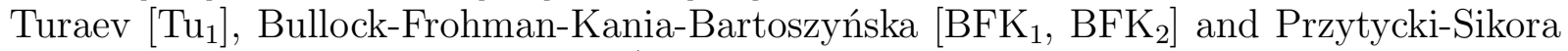
$[\mathrm{PrS}]$ showed that the skein algebra $\mathcal{S}^{A}(S)$ provides a quantization of the character variety

$$
\mathcal{R}_{\mathrm{SL}_{2}(\mathbb{C})}(S)=\left\{\text { group homomorphisms } r: \pi_{1}(S) \rightarrow \mathrm{SL}_{2}(\mathbb{C})\right\} / / \mathrm{SL}_{2}(\mathbb{C})
$$

where $\mathrm{SL}_{2}(\mathbb{C})$ acts on homomorphisms by conjugation, and where the double bar indicates that the quotient is to be taken in the sense of geometric invariant theory [MFK]. In fact, if

Date: September 24, 2018.

This research was partially supported by grants DMS-1105402, DMS-1105692, DMS-1406559 from the U.S. National Science Foundation. In addition, the article was extensively rewritten and reorganized while the first author was a Simons Fellow (grant 301050 from the Simons Foundation) in 2014-15, as well as a Simons Visiting Professor at the Mathematical Sciences Research Institute in Berkeley, California, (NSF grant 09032078000) in the Spring 2015 semester. 


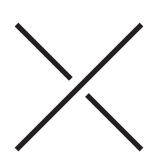

$K_{1}$
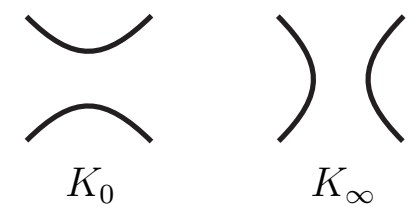

Figure 1. A Kauffman triple

one follows the physical tradition that a quantization of a space $X$ replaces the commutative algebra of functions on $X$ by a non-commutative algebra of operators on a Hilbert space, an element of the quantization should be a representation of the skein algebra.

When $A$ is a root of unity, a classical example of a finite-dimensional representation of the skein algebra $\mathcal{S}^{A}(S)$ arises from the Witten-Reshetikhin-Turaev topological quantum field theory associated to the fundamental representation of the quantum group $\mathrm{U}_{q}\left(\mathfrak{s l}_{2}\right)$ Wit, ReT, BHMV, $\mathrm{Tu}_{2}, \mathrm{BoW}_{5}$. The main purpose of the current article is to provide a wider family of such representations when the surface $S$ has at least one puncture. The case of closed surfaces is considered in the subsequent article $\mathrm{BoW}_{4}$, which builds on this one.

In $\left[\mathrm{BoW}_{3}\right.$, we identified invariants for irreducible finite-dimensional representations $\rho$ : $\mathcal{S}^{A}(S) \rightarrow \operatorname{End}(E)$, in the case where $A^{2}$ is a primitive $N$-root of unity with $N$ odd. These invariants are a little easier to describe when $A^{N}=-1$, and most of the current article will be devoted to this case. We indicate in $\$ 6$ how the other possible case when $A^{N}=+1$ can be deduced from this one. Because $N$ is odd, the property that $A^{2}$ is a primitive $N$-root of unity with $A^{N}=-1$ is equivalent to the property that $A$ is a primitive $N$-root of -1 .

When $A^{N}=-1$, our main invariant is a point of the character variety $\mathcal{R}_{\mathrm{SL}_{2}(\mathbb{C})}(S)$. Its definition involves the $n$-th normalized Chebyshev polynomial $T_{n}(x)$ of the first kind, determined by the trigonometric identity that $2 \cos n \theta=T_{n}(2 \cos \theta)$. Equivalently, $\operatorname{Tr} M^{n}=T_{n}(\operatorname{Tr} M)$ for every matrix $M \in \mathrm{SL}_{2}(\mathbb{C})$.

A character $r \in \mathcal{R}_{\mathrm{SL}_{2}(\mathbb{C})}(S)$ associates a trace $\operatorname{Tr} r(K) \in \mathbb{C}$ to each closed curve $K$ on the surface $S$. This trace is independent of the homomorphism $\pi_{1}(S) \rightarrow \mathrm{SL}_{2}(\mathbb{C})$ used to represent $r$, or of the representative chosen in the conjugacy class of $\pi_{1}(S)$ representing $K$. In fact, the character variety $\mathcal{R}_{\mathrm{SL}_{2}(\mathbb{C})}(S)$ is defined in such a way that two homomorphisms $r: \pi_{1}(S) \rightarrow \mathrm{SL}_{2}(\mathbb{C})$ correspond to the same character if and only if they induce the same trace function $K \mapsto \operatorname{Tr} r(K)$.

Theorem $1\left(\left[\mathrm{BoW}_{3}\right]\right)$. Suppose that $A$ is a primitive $N$-root of -1 with $N$ odd, and let $\rho: \mathcal{S}^{A}(S) \rightarrow \operatorname{End}(E)$ be an irreducible finite-dimensional representation of the Kauffman bracket skein algebra. Let $T_{N}(x)$ be the $N$-th normalized Chebyshev polynomial of the first kind.

(1) There exists a unique character $r_{\rho} \in \mathcal{R}_{\mathrm{SL}_{2}(\mathbb{C})}(S)$ such that

$$
T_{N}(\rho([K]))=-\left(\operatorname{Tr} r_{\rho}(K)\right) \operatorname{Id}_{E}
$$

for every framed knot $K \subset S \times[0,1]$ whose projection to $S$ has no crossing and whose framing is vertical.

(2) Let $P_{k}$ be a small simple loop going around the $k$-th puncture of $S$, and consider it as a knot in $S \times[0,1]$ with vertical framing. Then there exists a number $p_{k} \in \mathbb{C}$ such that $\rho\left(\left[P_{k}\right]\right)=p_{k} \operatorname{Id}_{E}$.

(3) The number $p_{k}$ of $(2)$ is related to the character $r_{\rho} \in \mathcal{R}_{\mathrm{SL}_{2}(\mathbb{C})}(S)$ of $(1)$ by the property that $T_{N}\left(p_{k}\right)=-\operatorname{Tr} r_{\rho}\left(P_{k}\right)$. 
The character $r_{\rho} \in \mathcal{R}_{\mathrm{SL}_{2}(\mathbb{C})}(S)$ associated to the irreducible representation $\rho: \mathcal{S}^{A}(S) \rightarrow$ End $(E)$ by Part (1) of Theorem 1 is the classical shadow of $\rho$. This Part (1) also admits a generalization to all framed links $K \subset S \times[0,1]$, which involves the element $\left[K^{T_{N}}\right] \in \mathcal{S}^{A}(S)$ defined by threading the Chebyshev polynomial $T_{N}$ along all components of $K$; see $\mathrm{BoW}_{3}$ for a precise statement. The numbers $p_{k}$ defined by Part (2) are the puncture invariants of the representation $\rho$. Part (3) shows that, once the classical shadow $r_{\rho}$ is known, there are at most $N$ possible values for each of the puncture invariants $p_{k}$.

The classical shadow provides one more example of a situation where a quantum object determines one of the classical objects that are being quantized. See also [Lê] for a different and more recent approach to the key results underlying Theorem 1.

The main result of this article is the following converse statement.

Theorem 2. Assume that the surface $S$ has at least one puncture, that its Euler characteristic is negative, that $A$ is a primitive $N$-root of -1 with $N$ odd, and that we are given:

(1) a character $r \in \mathcal{R}_{\mathrm{SL}_{2}(\mathbb{C})}(S)$ which realizes some ideal triangulation of $S$ in the sense discussed in 93 ;

(2) a number $p_{k} \in \mathbb{C}$ such that $T_{N}\left(p_{k}\right)=-\operatorname{Tr} r\left(P_{k}\right)$ for each of the punctures of $S$.

Then, there exists an irreducible finite-dimensional representation $\rho: \mathcal{S}^{A}(S) \rightarrow \operatorname{End}(E)$ whose classical shadow is equal to $r$ and whose puncture invariants are the $p_{k}$.

The requirement that $r$ realizes some ideal representation is fairly mild. It can be shown to be satisfied by all points outside of an algebraic subset of complex codimension $2|\chi(S)|-1$ in the character variety $\mathcal{R}_{\mathrm{SL}_{2}(\mathbb{C})}(S)$.

The sequel $\left[\mathrm{BoW}_{4}, \overline{\mathrm{BoW}_{6}}\right.$ to this paper greatly improves Theorem 2, In particular, it removes the requirements that $r$ realizes an ideal triangulation, and that $S$ has at least one puncture. It also shows that the representation provided by our construction is independent of the many choices made during the argument, so that its output is natural, in particular with respect to the action of the mapping class group $\pi_{0} \operatorname{Diff}(S)$ of the surface. The constructions and results of the current article are a key ingredient in the proofs of $\mathrm{BoW}_{4}$ and $\mathrm{BoW}_{6}$.

The proof of Theorem 2 uses as a fundamental tool the quantum trace homomorphism $\operatorname{Tr}_{\lambda}^{\omega}: \mathcal{S}^{A}(S) \rightarrow \mathcal{T}^{\omega}(\lambda)$, constructed in $\left[\mathrm{BoW}_{1}\right.$, which embeds the skein algebra in the quantum Teichmüller space. The quantum Teichmüller space is here incarnated as the ChekhovFock algebra $\mathcal{T}^{\omega}(\lambda)$ of an ideal triangulation $\lambda$ of the surface, and is a quantization of an object that is closely related to the character variety $\mathcal{R}_{\mathrm{SL}_{2}(\mathbb{C})}(S)$. It is not as natural as the Kauffman bracket skein algebra, but its algebraic structure is very simple. In particular, its representation theory is relatively easy to analyze [BoL]. The same holds for a smaller algebra $Z^{\omega}(\lambda) \subset \mathcal{T}^{\omega}(\lambda)$ containing the image of the quantum trace homomorphism $\operatorname{Tr}_{\lambda}^{\omega}$. Composing representations of $\mathcal{Z}^{\omega}(\lambda)$ with the homomorphism $\operatorname{Tr}_{\lambda}^{\omega}: \mathcal{S}^{A}(S) \rightarrow Z^{\omega}(\lambda)$ provides an extensive family of representations of the skein algebra $\mathcal{S}^{A}(S)$, which can then be used to prove Theorem 2 .

The main technical challenge in this strategy is to compute the classical shadow of the representations of $\mathcal{S}^{A}(S)$ so obtained, in terms of the parameters controlling the original representations of $z^{\omega}(\lambda)$. This is provided by the miraculous cancellations discovered in $\left[\mathrm{BoW}_{3}\right.$. These properties show that the quantum trace homomorphism $\operatorname{Tr}_{\lambda}^{\omega}: \mathcal{S}^{A}(S) \rightarrow Z^{\omega}(\lambda)$ is well-behaved with respect to the Chebyshev homomorphism $\mathcal{S}^{-1}(S) \rightarrow \mathcal{S}^{A}(S)$ used to 
define the classical shadow of a representation of $\mathcal{S}^{A}(S)$, and with respect to the Frobenius homomorphism $Z^{\iota}(\lambda) \rightarrow Z^{\omega}(\lambda)$ which computes the invariants of representations of $Z^{\omega}(\lambda)$.

One of the steps in the proof, used to determine the algebraic structure of the algebra $Z^{\omega}(\lambda)$, may be of interest by itself. This statement describes the structure of the Thurston intersection form on the set $\mathcal{W}(\tau ; \mathbb{Z})$ of integer-valued edge weight systems for a train track $\tau$. The result is well-known for real-valued weights. However, the integer valued case has subtler number-theoretic properties, resulting in the unexpected simultaneous occurrence of blocks $\left(\begin{array}{cc}0 & 1 \\ -1 & 0\end{array}\right)$ and $\left(\begin{array}{cc}0 & 2 \\ -2 & 0\end{array}\right)$ in the block diagonalization of the Thurston form. See Theorem 26 in the Appendix. Because of the ubiquity of the Thurston intersection form in many geometric problems (for instance the relationship between complex lengths and the shear-bend cocycle $\beta \in \mathcal{W}(\tau ; \mathbb{C} / 2 \pi \mathrm{i} \mathbb{Z})$ of a pleated surface $[\mathrm{Bo}])$, this statement is probably of interest beyond the quantum topology scope of the current article.

See the recent preprints $\mathrm{AbF}_{1}, \mathrm{AbF}_{2}$ for another construction of representations of $\mathcal{S}^{A}(S)$ with a given classical shadow $r \in \mathcal{R}_{\mathrm{SL}_{2}(\mathbb{C})}(S)$, valid for $r$ in a Zariski dense open subset of $\mathcal{R}_{\mathrm{SL}_{2}(\mathbb{C})}(S)$. The construction of $\mathrm{AbF}_{1}, \mathrm{AbF}_{2}$ is simpler, but ours is more explicit. In the few cases where the dimension of the representations of $\mathrm{AbF}_{1}, \mathrm{AbF}_{2}$ can be computed, these dimensions are significantly larger than those arising in the current article.

\section{The Chekhov-Fock algebra And the quantum trace homomorphism}

1.1. The Chekhov-Fock algebra. The Chekhov-Fock algebra (introduced in BoL as a reinterpretation of key insights from $\left.\mathrm{ChF}_{1}, \mathrm{ChF}_{2}, \mathrm{Fo}\right]$ ) is the avatar of the quantum Teichmüller space associated to an ideal triangulation of the surface $S$. See also $\mathrm{Ka}$ ] for a related construction, and [BoL, $\mathrm{Liu}]$ for more discussion.

If $S$ is obtained from a compact surface $\bar{S}$ by removing finitely many points $v_{1}, v_{2}, \ldots, v_{s}$, an ideal triangulation of $S$ is a triangulation $\lambda$ of $\bar{S}$ whose vertex set is exactly $\left\{v_{1}, v_{2}, \ldots, v_{s}\right\}$. The surface $S$ admits an ideal triangulation if and only if it is non-compact and if its Euler characteristic is negative; we will consequently assume these properties satisfied throughout the article. If the surface has genus $g$ and $s$ punctures, an ideal triangulation then has $n=6 g+3 s-6$ edges and $4 g+2 s-4$ faces.

Let $e_{1}, e_{2}, \ldots, e_{n}$ denote the edges of $\lambda$. Let $a_{i} \in\{0,1,2\}$ be the number of times an end of the edge $e_{j}$ immediately succeeds an end of $e_{i}$ when going counterclockwise around a puncture of $S$, and set $\sigma_{i j}=a_{i j}-a_{j i} \in\{-2,-1,0,1,2\}$. The Chekhov-Fock algebra $\mathcal{T}^{\omega}(\lambda)$ of $\lambda$ is the algebra defined by generators $Z_{1}^{ \pm 1}, Z_{2}^{ \pm 1}, \ldots, Z_{n}^{ \pm 1}$ associated to the edges $e_{1}, e_{2}$, $\ldots, e_{n}$ of $\lambda$, and by the relations

$$
Z_{i} Z_{j}=\omega^{2 \sigma_{i j}} Z_{j} Z_{i}
$$

Remark 3. The actual Chekhov-Fock algebra $\mathcal{T}^{q}(\lambda)$ that is at the basis of the quantum Teichmüller space uses the constant $q=\omega^{4}$ instead of $\omega$. The generators $Z_{i}$ of $\mathcal{T}^{\omega}(\lambda)$ appearing here are designed to model square roots of the original generators of $\mathcal{T}^{q}(\lambda)$.

An element of the Chekhov-Fock algebra $\mathcal{T}^{\omega}(\lambda)$ is a linear combination of monomials $Z_{i_{1}}^{n_{1}} Z_{i_{2}}^{n_{2}} \ldots Z_{i_{l}}^{n_{l}}$ in the generators $Z_{i}$, with $n_{1}, n_{2}, \ldots, n_{l} \in \mathbb{Z}$. Because of the skew-commutativity relation $Z_{i} Z_{j}=\omega^{2 \sigma_{i j}} Z_{j} Z_{i}$, the order of the variables in such a monomial does matter. It is convenient to use the following symmetrization trick. The Weyl quantum ordering for $Z_{i_{1}}^{n_{1}} Z_{i_{2}}^{n_{2}} \ldots Z_{i_{l}}^{n_{l}}$ is the monomial

$$
\left[Z_{i_{1}}^{n_{1}} Z_{i_{2}}^{n_{2}} \ldots Z_{i_{l}}^{n_{l}}\right]=\omega^{-\sum_{u<v} n_{u} n_{v} \sigma_{i_{u} i_{v}}} Z_{i_{1}}^{n_{1}} Z_{i_{2}}^{n_{2}} \ldots Z_{i_{l}}^{n_{l}} .
$$


The formula is specially designed that $\left[Z_{i_{1}}^{n_{1}} Z_{i_{2}}^{n_{2}} \ldots Z_{i_{l}}^{n_{l}}\right]$ is invariant under any permutation of the $Z_{i_{u}}^{n_{u}}$. Note that the algebraic structure of the Chekhov-Fock algebra $\mathcal{T}^{\omega}(\lambda)$ depends only on the square $\omega^{2}$, but that the Weyl quantum ordering depends on the choice of $\omega$.

\subsection{The quantum trace homomorphism.}

Theorem $4\left(\left[\mathrm{BoW}_{1}\right)\right.$. For $A=\omega^{-2}$, there exists an injective algebra homomorphism

$$
\operatorname{Tr}_{\lambda}^{\omega}: \mathcal{S}^{A}(S) \rightarrow \mathcal{T}^{\omega}(\lambda)
$$

The specific homomorphism $\operatorname{Tr}_{\lambda}^{\omega}$ constructed in $\left[\mathrm{BoW}_{1}\right.$ is the quantum trace homomorphism. It is uniquely determined by certain properties stated in that article, but we will only need to know that it exists and that it satisfies the properties given in $\$ 1.3$ below.

1.3. The Chebyshev and Frobenius homomorphisms. We now assume that $A$ is a primitive $N$-root of -1 with $N$ odd. Recall that $T_{N}$ denotes the $N$-th normalized Chebyshev polynomial, defined by the property that $\cos N \theta=\frac{1}{2} T_{N}(2 \cos \theta)$ for every $\theta$.

Theorem $5\left(\mathrm{BoW}_{3}\right)$. When $A$ is a primitive $N$-root of -1 with $N$ odd, there is a unique algebra homomorphism $\mathbf{T}^{A}: \mathcal{S}^{-1}(S) \rightarrow \mathcal{S}^{A}(S)$ such that

$$
\mathbf{T}^{A}([K])=T_{N}([K])
$$

for every framed knot $K \subset S \times[0,1]$ whose projection to $S$ has no crossing and whose framing is vertical. In addition, the image of $\mathbf{T}^{A}$ is central in $\mathcal{S}^{A}(S)$.

The homomorphism $\mathbf{T}^{A}$ provided by Proposition 5 is the Chebyshev homomorphism. It is a key ingredient in the definition of the invariants of Theorem 1 .

There is an analogous and much simpler homomorphism at the level of the Chekhov-Fock algebra, namely the following Frobenius homomorphism.

Proposition 6. If $\iota=\omega^{N^{2}}$, there is an algebra homomorphism

$$
\mathbf{F}^{\omega}: \mathcal{T}^{\iota}(\lambda) \rightarrow \mathcal{T}^{\omega}(\lambda)
$$

which maps each generator $Z_{i} \in \mathcal{T}^{\iota}(\lambda)$ to $Z_{i}^{N} \in \mathcal{T}^{\omega}(\lambda)$, where in the first instance $Z_{i} \in \mathcal{T}^{\iota}(\lambda)$ denotes the generator associated to the $i$-th edge $e_{i}$ of $\lambda$, whereas the second time $Z_{i} \in \mathcal{T}^{\omega}(\lambda)$ denotes the generator of $\mathcal{T}^{\omega}(\lambda)$ associated to the same edge $e_{i}$.

Note that $\iota^{2}=\omega^{2 N^{2}}=A^{-N^{2}}=(-1)^{N}=-1$, so that $\iota= \pm \mathrm{i}$.

The following compatibility statement, which connects the Chebyshev homomorphism to the Frobenius homomorphism through appropriate quantum trace homomorphisms, is fundamental for our arguments. This result encapsulates the miraculous cancellations of $\mathrm{BoW}_{3}$.

Theorem $7\left(\mathrm{BoW}_{3}\right)$. The diagram

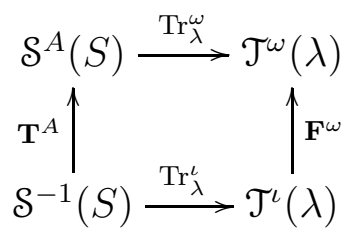

is commutative. Namely, for every skein $[K] \in \mathcal{S}^{-1}(S)$, the quantum trace $\operatorname{Tr}_{\lambda}^{\omega}\left(\mathbf{T}^{A}([K])\right)$ of $\mathbf{T}^{A}([K])$ is obtained from the classical trace polynomial $\operatorname{Tr}_{\lambda}^{\iota}([K])$ by replacing each generator $Z_{i} \in \mathcal{T}^{\iota}(\lambda)$ by $Z_{i}^{N} \in \mathcal{T}^{\omega}(\lambda)$. 


\section{The Balanced Chekhov-Fock algebra}

2.1. The balanced Chekhov-Fock algebra. The quantum trace homomorphism $\operatorname{Tr}_{\lambda}^{\omega}$ of Theorem 4 (and $\left[\mathrm{BoW}_{1}\right]$ ) is far from being surjective. Indeed, for a skein $[K] \in \mathcal{S}^{A}(S)$ represented by a framed link $K \subset S \times[0,1]$, the exponents of the monomials $Z_{1}^{k_{1}} Z_{2}^{k_{2}} \ldots Z_{n}^{k_{n}}$ appearing in the expression of $\operatorname{Tr}_{\lambda}^{\omega}([K])$ are balanced, in the sense that they satisfy the following parity condition: for every triangle $T_{j}$ of the ideal triangulation $\lambda$, the sum $k_{i_{1}}+$ $k_{i_{2}}+k_{i_{3}}$ of the exponents of the generators $Z_{i_{1}}, Z_{i_{2}}, Z_{i_{3}}$ associated to the sides of $T_{j}$ is even.

Let $Z^{\omega}(\lambda)$ denote the sub-algebra of $\mathcal{T}^{\omega}(\lambda)$ generated by all monomials satisfying this exponent parity condition. By definition, $z^{\omega}(\lambda)$ is the balanced Chekhov-Fock algebra of the ideal triangulation $\lambda$. It is designed so that the quantum trace homomorphism restricts to a homomorphism $\operatorname{Tr}_{\lambda}^{\omega}: \mathcal{S}^{A}(S) \rightarrow \mathcal{Z}^{\omega}(\lambda)$.

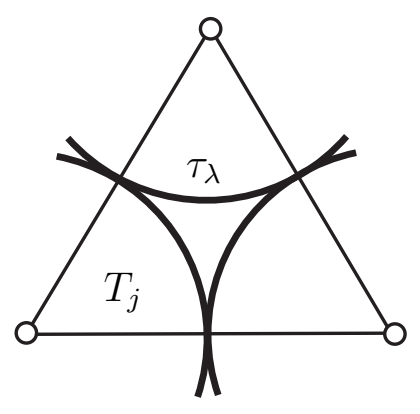

FIGURE 2.

To keep track of the exponent parity condition defining the monomials of $z^{\omega}(\lambda)$, it is convenient to consider a train track $\tau_{\lambda}$ which, on each triangle $T_{j}$ of the ideal triangulation $\lambda$, looks as in Figure 2. In particular, $\tau_{\lambda}$ has one switch for each edge of $\lambda$, and three edges for each triangle of $\lambda$. Let $\mathcal{W}\left(\tau_{\lambda} ; \mathbb{Z}\right)$ be the set of integer edge weight systems $\alpha$ for $\tau_{\lambda}$, assigning a number $\alpha(e) \in \mathbb{Z}$ to each edge $e$ of $\tau_{\lambda}$ in such a way that, at each switch, the weights of the edges incoming on one side add up to the sum of the weights of the edges outgoing on the other side. There is a natural map $\mathcal{W}\left(\tau_{\lambda} ; \mathbb{Z}\right) \rightarrow \mathbb{Z}^{n}$ which, given an edge weight system, associates to each of the $n$ switches of $\tau_{\lambda}$ the sum of the weights of the edges incoming on any side of the switch. Then, an element $\left(k_{1}, k_{2}, \ldots, k_{n}\right) \in \mathbb{Z}^{n}$ satisfies the above parity condition if and only if it is in the image of this map. Also, the map $\mathcal{W}\left(\tau_{\lambda} ; \mathbb{Z}\right) \rightarrow \mathbb{Z}^{n}$ is easily seen to be injective. Since the image of this map has finite index, it follows that $\mathcal{W}\left(\tau_{\lambda} ; \mathbb{Z}\right)$ is isomorphic to $\mathbb{Z}^{n}$ as an abelian group.

This enables us to give a different description of $\mathcal{Z}^{\omega}(\lambda)$. For a weight system $\alpha \in \mathcal{W}\left(\tau_{\lambda} ; \mathbb{Z}\right)$, assigning a weight $\alpha_{i} \in \mathbb{Z}$ to the $i$-th edge $e_{i}$ of $\lambda$ (= the $i$-th switch of $\tau_{\lambda}$ ), define

$$
Z_{\alpha}=\left[Z_{1}^{\alpha_{1}} Z_{2}^{\alpha_{2}} \ldots Z_{n}^{\alpha_{n}}\right] \in Z^{\omega}(\lambda)
$$

where the bracket [ ] denotes the Weyl quantum ordering defined in $\$ 1.1$.

The above discussion proves the following fact.

Lemma 8. As $\alpha \in \mathcal{W}\left(\tau_{\lambda} ; \mathbb{Z}\right)$ ranges over all weight systems for the train track $\tau_{\lambda}$, the associated monomials $Z_{\alpha}$ form a basis for the vector space $Z^{\omega}(\lambda)$.

We can elaborate a little on the structure of the group $\mathcal{W}\left(\tau_{\lambda} ; \mathbb{Z}\right)$. By definition of the parity condition, $\mathcal{W}\left(\tau_{\lambda} ; \mathbb{Z}\right) \subset \mathbb{Z}^{n}$ contains the subset $(2 \mathbb{Z})^{n}$ consisting of all switch weight systems $\left(\alpha_{1}, \alpha_{2}, \ldots, \alpha_{n}\right) \in \mathbb{Z}^{n}$ where the $\alpha_{i}$ are even. Also, given $\alpha \in \mathcal{W}\left(\tau_{\lambda} ; \mathbb{Z}\right)$, we can define 
a chain with coefficients in $\mathbb{Z}_{2}$ by endowing each edge $e$ of the train track $\tau_{\lambda}$ with the modulo 2 reduction of the weight $\alpha(e) \in \mathbb{Z}$. The switch relations guarantee that this chain is closed, and this defines a natural homomorphism $\mathcal{W}\left(\tau_{\lambda} ; \mathbb{Z}\right) \rightarrow H_{1}\left(S ; \mathbb{Z}_{2}\right)$.

Lemma 9. The inclusion map and homomorphism above define an exact sequence

$$
0 \rightarrow(2 \mathbb{Z})^{n} \rightarrow \mathcal{W}\left(\tau_{\lambda} ; \mathbb{Z}\right) \rightarrow H_{1}\left(S ; \mathbb{Z}_{2}\right) \rightarrow 0 .
$$

Proof. The homomorphism $\mathcal{W}\left(\tau_{\lambda} ; \mathbb{Z}\right) \rightarrow H_{1}\left(S ; \mathbb{Z}_{2}\right)$ can also be expressed in terms of the dual graph $\Gamma_{\lambda}$ of the triangulation $\lambda$. Indeed, the class $[\alpha] \in H_{1}\left(S ; \mathbb{Z}_{2}\right)$ induced by $\alpha \in \mathcal{W}\left(\tau_{\lambda} ; \mathbb{Z}\right)$ is also realized by endowing each edge $f_{i}$ of $\Gamma_{\lambda}$ with the modulo 2 reduction of the switch weight $\alpha_{i}$ associated by $\alpha$ to the edge $e_{i}$ of $\lambda$ that is dual to $f_{i}$; the parity condition guarantees that this chain is really closed. The result then immediately follows from the definitions, and from the isomorphism $H_{1}\left(\Gamma_{\lambda} ; \mathbb{Z}_{2}\right) \cong H_{1}\left(S ; \mathbb{Z}_{2}\right)$ coming from the fact that the surface $S$ deformation retracts to the dual graph $\Gamma_{\lambda}$.

Note that the exact sequence of Lemma 9 admits no partial splitting.

2.2. The algebraic structure of the balanced Chekhov-Fock algebra. We first describe the multiplicative structure of the balanced Chekhov-Fock algebra $z^{\omega}(\lambda)$ in the context of Lemma 8 .

The space $\mathcal{W}\left(\tau_{\lambda} ; \mathbb{Z}\right)$ carries a very natural antisymmetric bilinear form

$$
\Omega: \mathcal{W}\left(\tau_{\lambda} ; \mathbb{Z}\right) \times \mathcal{W}\left(\tau_{\lambda} ; \mathbb{Z}\right) \rightarrow \mathbb{Z}
$$

the Thurston intersection form defined by the property that, for $\alpha, \beta \in \mathcal{W}\left(\tau_{\lambda} ; \mathbb{Z}\right)$,

$$
\Omega(\alpha, \beta)=\frac{1}{2} \sum_{e \text { right of } e^{\prime}}\left(\alpha(e) \beta\left(e^{\prime}\right)-\alpha\left(e^{\prime}\right) \beta(e)\right)
$$

where the sum is over all pairs $\left(e, e^{\prime}\right)$ of edges of $\tau_{\lambda}$ such that $e$ and $e^{\prime}$ come out of the same side of some switch of $\tau_{\lambda}$, with $e$ to the right of $e^{\prime}$. See Lemma 28 in the Appendix for a more conceptual interpretation of $\Omega$, and for a proof that $\Omega(\alpha, \beta)$ is really an integer.

Lemma 10. For every $\alpha, \beta \in \mathcal{W}\left(\tau_{\lambda} ; \mathbb{Z}\right)$,

$$
Z_{\alpha} Z_{\beta}=\omega^{2 \Omega(\alpha, \beta)} Z_{\alpha+\beta}
$$

In particular, $Z_{\alpha} Z_{\beta}=\omega^{4 \Omega(\alpha, \beta)} Z_{\beta} Z_{\alpha}$.

Proof. The second statement, that $Z_{\alpha} Z_{\beta}=\omega^{4 \Omega(\alpha, \beta)} Z_{\beta} Z_{\alpha}$, is a simple computation. After observing that this property holds for any $\omega$ (not just roots of unity), the first statement, that $Z_{\alpha} Z_{\beta}=\omega^{2 \Omega(\alpha, \beta)} Z_{\alpha+\beta}$, then follows by definition of the Weyl quantum ordering.

This is particularly simple if we replace $\omega$ by $\iota=\omega^{N^{2}}$, with the assumption that $A^{2 N}=1$ so that $\iota^{4}=\omega^{4 N^{2}}=A^{-2 N^{2}}=1$.

Corollary 11. If $\iota^{4}=1$, the algebra $z^{\iota}(\lambda)$ is commutative.

In general, the key to understanding the algebraic structure of $z^{\omega}(\lambda)$ is Lemma 12 below.

For $k=1, \ldots, s$, the $k$-th puncture of $S$ specifies an element $\eta_{k} \in \mathcal{W}\left(\tau_{\lambda} ; \mathbb{Z}\right)$ defined as follows: For every edge $e$ of $\tau_{\lambda}$, the edge weight $\eta_{k}(e) \in\{0,1,2\}$ is the number of sides of $e$ that are adjacent to the same component of $S-\tau_{\lambda}$ as this puncture.

Recall that the surface $S$ has genus $g$ and $s$ punctures. 
Lemma 12. The lattice $\mathcal{W}\left(\tau_{\lambda} ; \mathbb{Z}\right) \cong \mathbb{Z}^{n}$ admits a basis in which the matrix of the Thurston intersection form $\Omega$ is block diagonal with $g$ blocks $\left(\begin{array}{cc}0 & 1 \\ -1 & 0\end{array}\right), 2 g+s-3$ blocks $\left(\begin{array}{cc}0 & 2 \\ -2 & 0\end{array}\right)$ and $s$ blocks $(0)$. In addition, the kernel of $\Omega$ is freely generated by the elements $\eta_{1}, \eta_{2}, \ldots$, $\eta_{s} \in \mathcal{W}\left(\tau_{\lambda} ; \mathbb{Z}\right)$ associated to the punctures of $S$ as above.

Proof. This is a special case of a result given by Theorem 26 in the Appendix, which determines the algebraic structure of the Thurston intersection form for a general train track $\tau$. When applying this result to the train track $\tau_{\lambda}$, the numbers $h, n_{\text {even }}$ and $n_{\text {odd }}$ of Theorem 26 are respectively equal to the genus $g$ of the surface $S$, to the number $s$ of punctures of $S$, and to the number $4 g+2 s-4$ of triangles of the ideal triangulation $\lambda$.

The combination of Lemmas 8, 10 and 12 now provides the complete algebraic structure of the balanced Chekhov-Fock algebra $\mathcal{Z}^{\omega}(\lambda)$. Let $\mathcal{W}^{q}$ denote the algebra, known as the quantum 2-torus, defined by generators $X^{ \pm 1}, Y^{ \pm 1}$ and by the relation $X Y=q Y X$.

Corollary 13. For $q=\omega^{4}$, the balanced Chekhov-Fock algebra $z^{\omega}(\lambda)$ is isomorphic to

$$
\mathcal{W}_{1}^{q} \otimes \mathcal{W}_{2}^{q} \otimes \cdots \otimes \mathcal{W}_{g}^{q} \otimes \mathcal{W}_{g+1}^{q^{2}} \otimes \mathcal{W}_{g+2}^{q^{2}} \otimes \cdots \otimes \mathcal{W}_{3 g+s-3}^{q^{2}} \otimes \mathbb{C}\left[H_{1}\right] \otimes \mathbb{C}\left[H_{2}\right] \otimes \cdots \otimes \mathbb{C}\left[H_{s}\right]
$$

where each $\mathcal{W}_{i}^{q}$ is a copy of the quantum 2 -torus $\mathcal{W}^{q}$, each $\mathcal{W}_{j}^{q^{2}}$ is a copy of $\mathcal{W}^{q^{2}}$, and each $\mathbb{C}\left[H_{k}\right]$ is a polynomial algebra in the variable $H_{k}$.

In addition, the $s$ central generators $H_{k}=Z_{\eta_{k}}$ are associated to the punctures of $S$ as in Lemma 12 .

2.3. Representations of the balanced Chekhov-Fock algebra. The algebraic structure of the balanced Chekhov-Fock algebra $Z^{\omega}(\lambda)$ determined in Corollary 13 is relatively simple. This makes it easy to classify its irreducible finite-dimensional representations.

As usual, we assume that $A=\omega^{-2}$ is a primitive $N$-root of -1 , with $N$ odd.

Proposition 14. Let $\mu: Z^{\omega}(\lambda) \rightarrow \operatorname{End}(E)$ be an irreducible finite-dimensional representation of $Z^{\omega}(\lambda)$. There exists a map $\zeta_{\mu}: \mathcal{W}\left(\tau_{\lambda} ; \mathbb{Z}\right) \rightarrow \mathbb{C}^{*}$ and numbers $h_{k} \in \mathbb{C}^{*}$, with $k=1, \ldots, s$, associated to the punctures of the surface $S$ such that:

(1) $\mu\left(Z_{\alpha}^{N}\right)=\zeta_{\mu}(\alpha) \operatorname{Id}_{E}$ for every edge weight system $\alpha \in \mathcal{W}\left(\tau_{\lambda} ; \mathbb{Z}\right)$ with associated monomial $Z_{\alpha} \in Z^{\omega}(\lambda)$

(2) $\zeta_{\mu}(\alpha+\beta)=(-1)^{\Omega(\alpha, \beta)} \zeta_{\mu}(\alpha) \zeta_{\mu}(\beta)$ for every $\alpha, \beta \in \mathcal{W}\left(\tau_{\lambda} ; \mathbb{Z}\right)$, where $\Omega$ is the Thurston intersection form;

(3) $\mu\left(H_{k}\right)=h_{k} \operatorname{Id}_{E}$ for the central element $H_{k}=Z_{\eta_{k}} \in z^{\omega}(\lambda)$ associated to the $k$-th puncture of $S$ as in Corollary 13;

(4) $\zeta_{\mu}\left(\eta_{k}\right)=h_{k}^{N}$ for the weight system $\eta_{k} \in \mathcal{W}\left(\tau_{\lambda} ; \mathbb{Z}\right)$ associated to the $k$-th puncture of $S$ as in Lemma 12 .

Proof. For every $\alpha \in \mathcal{W}\left(\tau_{\lambda} ; \mathbb{Z}\right)$, Lemma 10 shows that the element $Z_{\alpha}^{N}=Z_{N \alpha}$ is central in $z^{\omega}(\lambda)$. In particular, if $\mu: Z^{\omega}(\lambda) \rightarrow \operatorname{End}(E)$ is an irreducible finite-dimensional representation of $z^{\omega}(\lambda)$, there is a number $\zeta_{\mu}(\alpha) \in \mathbb{C}^{*}$ such that $\mu\left(Z_{\alpha}^{N}\right)=\zeta_{\mu}(\alpha) \operatorname{Id}_{E}$. In addition, Lemma 10 shows that $Z_{\alpha}^{N} Z_{\beta}^{N}=\omega^{2 N^{2} \Omega(\alpha, \beta)} Z_{\alpha+\beta}^{N}=(-1)^{\Omega(\alpha, \beta)} Z_{\alpha+\beta}^{N}$, so that the map $\zeta_{\mu}: \mathcal{W}\left(\tau_{\lambda} ; \mathbb{Z}\right) \rightarrow \mathbb{C}^{*}$ satisfies Property $(2)$

Similarly, Corollary 13 shows that each $H_{k}$ is central in $z^{\omega}(\lambda)$, so that $\mu\left(H_{k}\right)=h_{k} \operatorname{Id}$ for some $h_{k} \in \mathbb{C}^{*}$. Then $h_{k}^{N} \operatorname{Id}_{E}=\mu\left(H_{k}^{N}\right)=\mu\left(Z_{\eta_{k}}^{N}\right)=\zeta_{\mu}\left(\eta_{k}\right) \operatorname{Id}_{E}$ since $H_{k}=Z_{\eta_{k}}$, so that $\zeta_{\mu}\left(\eta_{k}\right)=h_{k}^{N}$. 
A map $\zeta: \mathcal{W}\left(\tau_{\lambda} ; \mathbb{Z}\right) \rightarrow \mathbb{C}^{*}$ satisfying Condition (2) of Proposition 14 is a twisted homomorphism twisted by the Thurston form $\Omega$, or more precisely twisted by the symmetric map $(\alpha, \beta) \mapsto(-1)^{\Omega(\alpha, \beta)}$. This notion will probably look less intimidating once one realizes that a twisted homomorphism is completely determined by the assignment of a non-zero complex number to each of the $n$ generators of the group $\mathcal{W}\left(\tau_{\lambda} ; \mathbb{Z}\right) \cong \mathbb{Z}^{n}$.

Proposition 15. Suppose that we are given a twisted homomorphism $\zeta: \mathcal{W}\left(\tau_{\lambda} ; \mathbb{Z}\right) \rightarrow \mathbb{C}^{*}$ twisted by the Thurston form $\Omega$ and, for each of the punctures of $S$, a number $h_{k} \in \mathbb{C}^{*}$ such that $h_{k}^{N}=\zeta\left(\eta_{k}\right)$. Then, up to isomorphism, there exists a unique irreducible finitedimensional representation $\mu: z^{\omega}(\lambda) \rightarrow \operatorname{End}(E)$ such that

(1) $\zeta_{\mu}=\zeta$, namely $\mu\left(Z_{\alpha}^{N}\right)=\zeta(\alpha) \operatorname{Id}_{E}$ for every $\alpha \in \mathcal{W}\left(\tau_{\lambda} ; \mathbb{Z}\right)$;

(2) $\mu\left(H_{k}\right)=h_{k} \operatorname{Id}_{E}$ for $k=1, \ldots, s$.

In addition, for such a representation, the vector space E has dimension $N^{3 g+s-3}$.

Proof. Using elementary linear algebra, this is an immediate consequence of Corollary 13 , More precisely, consider the isomorphism

$$
\mathcal{Z}^{\omega}(\lambda) \cong \mathcal{W}_{1}^{q} \otimes \cdots \otimes \mathcal{W}_{g}^{q} \otimes \mathcal{W}_{g+1}^{q^{2}} \otimes \cdots \otimes \mathcal{W}_{3 g+s-3}^{q^{2}} \otimes \mathbb{C}\left[H_{1}\right] \otimes \cdots \otimes \mathbb{C}\left[H_{s}\right]
$$

provided by Corollary 13 .

For $1 \leqslant i \leqslant 3 g+s-3$, let $X_{i}^{ \pm 1}$ and $Y_{i}^{ \pm 1}$ denote the generators of $\mathcal{W}_{i}^{q}$ or $\mathcal{W}_{i}^{q^{2}}$ (satisfying the relation $X_{i} Y_{i}=q Y_{i} X_{i}$ if $1 \leqslant i \leqslant g$ and $X_{i} Y_{i}=q^{2} Y_{i} X_{i}$ if $\left.g<i \leqslant 3 g+s-3\right)$. The proof of Corollary 13 shows that these generators are of the form $X_{i}=Z_{\alpha_{i}}, Y_{i}=Z_{\beta_{i}}$ and $H_{k}=Z_{\eta_{k}}$ for some edge weight systems $\alpha_{i}, \beta_{i}, \eta_{k} \in \mathcal{W}\left(\tau_{\lambda} ; \mathbb{Z}\right)$. In addition, the $\alpha_{i}, \beta_{i}$ and $\eta_{k}$ form a basis for $\mathcal{W}\left(\tau_{\lambda} ; \mathbb{Z}\right) \cong \mathbb{Z}^{n}$.

Because $N$ is odd, $q=\omega^{4}$ and $q^{2}$ are both primitive $N$-root of unity. Arbitrarily pick $N-\operatorname{roots} \zeta\left(\alpha_{i}\right)^{\frac{1}{N}}$ and $\zeta\left(\beta_{i}\right)^{\frac{1}{N}}$, and define $\mu_{i}: \mathcal{W}_{i}^{q} \rightarrow \operatorname{End}\left(E_{i}\right)$ by the property that, if $v_{1}, v_{2}$, $\ldots, v_{N}$ form a basis for $E_{i} \cong \mathbb{C}^{N}$,

$$
\begin{aligned}
& \mu_{i}\left(X_{i}\right)\left(v_{j}\right)=-\zeta\left(\alpha_{i}\right)^{\frac{1}{N}} q^{j} v_{j} \text { and } \mu_{i}\left(Y_{i}\right)\left(v_{j}\right)=\zeta\left(\beta_{i}\right)^{\frac{1}{N}} v_{j+1} \text { if } 1 \leqslant i \leqslant g, \text { and } \\
& \mu_{i}\left(X_{i}\right)\left(v_{j}\right)=\zeta\left(\alpha_{i}\right)^{\frac{1}{N}} q^{2 j} v_{j} \text { and } \mu_{i}\left(Y_{i}\right)\left(v_{j}\right)=\zeta\left(\beta_{i}\right)^{\frac{1}{N}} v_{j+1} \text { if } g<i \leqslant 3 g+s-3 .
\end{aligned}
$$

Then, for $E=E_{1} \otimes E_{2} \otimes \cdots \otimes E_{3 g+s-3}$, define $\mu: Z^{\omega}(\lambda) \rightarrow \operatorname{End}(E)$ by the property that $\mu$ coincides with $\mu_{1} \otimes \mu_{2} \otimes \cdots \otimes \mu_{3 g+s-3}$ on $\mathcal{W}_{1}^{q} \otimes \mathcal{W}_{2}^{q} \otimes \cdots \otimes \mathcal{W}_{3 g+s-3}^{q}$, and $\mu\left(H_{k}\right)=h_{k} \operatorname{Id}_{E}$ for every $k=1, \ldots, s$.

It is immediate that $\mu$ satisfies the required properties. The fact that $\mu$ is irreducible, and that every irreducible representation is isomorphic to $\mu$, is easily proved by elementary linear algebra; see for instance [BoL, §4] for details.

\section{Pleated surfaces and homomorphisms to $\mathrm{SL}_{2}(\mathbb{C})$}

Let us consider the special case of Proposition [15] when $N=1$. In particular, $A=-1$ and $\iota=\omega= \pm \mathrm{i}$. Since the Chebyshev polynomial $T_{1}(x)$ is equal to $x$, the choice of puncture invariants $h_{k}$ is irrelevant and Proposition 15 associates to any twisted homomorphism $\zeta$ : $\mathcal{W}\left(\tau_{\lambda} ; \mathbb{Z}\right) \rightarrow \mathbb{C}^{*}$ a representation $\mu_{\zeta}: Z^{\iota}(\lambda) \rightarrow \operatorname{End}(\mathbb{C})$. By composition with the quantum trace homomorphism $\operatorname{Tr}_{\lambda}^{\iota}: \mathcal{S}^{-1}(S) \rightarrow \mathcal{Z}^{\iota}(\lambda)$ of Theorem 4 , we now have a homomorphism

$$
\rho_{\zeta}=\mu_{\zeta} \circ \operatorname{Tr}_{\lambda}^{\iota}: \mathcal{S}^{-1}(S) \rightarrow \operatorname{End}(\mathbb{C})=\mathbb{C}
$$

We can then apply the case $N=1$ of Theorem 1 (which actually is an observation of Doug Bullock, Charlie Frohman, Joanna Kania-Bartoszyńska, Jozef Przytycki and Adam Sikora 
$\left.\mathrm{Bu}_{1}, \mathrm{Bu}_{2}, \mathrm{BFK}_{1}, \mathrm{BFK}_{2}, \overline{\mathrm{PrS}}\right]$ and plays a crucial rôle in the proof of Theorem 1 in its full generality). It provides a character $r_{\zeta} \in \mathcal{R}_{\mathrm{SL}_{2}(\mathbb{C})}(S)$ such that

$$
\rho_{\zeta}([K])=-\operatorname{Tr} r_{\zeta}(K)
$$

for every framed knot $K \subset S \times[0,1]$. The property is valid for all knots, not just those whose projection to $S$ has no double point $\left[\mathrm{Bu}_{1}, \mathrm{Bu}_{2}, \mathrm{BFK}_{1}, \mathrm{BFK}_{2}, \mathrm{PrS}\right]$.

It is natural to ask which elements of $\mathcal{R}_{\mathrm{SL}_{2}(\mathbb{C})}(S)$ are obtained in this way. The answer involves the following geometric definition.

Let $\widetilde{S}$ be the universal cover of $S$, and let $\widetilde{\lambda}$ be the ideal triangulation of $\widetilde{S}$ obtained by lifting the edges and faces of $\lambda$. Identify $\mathrm{PSL}_{2}(\mathbb{C})$ to the isometry group of the hyperbolic 3 -space $\mathbb{H}^{3}$. A pleated surface with pleating locus $\lambda$ is the data $(\widetilde{f}, \bar{r})$ of a map $\widetilde{f}: \widetilde{S} \rightarrow \mathbb{H}^{3}$ and a group homomorphism $\bar{r}: \pi_{1}(S) \rightarrow \mathrm{PSL}_{2}(\mathbb{C})$ such that:

(1) $\tilde{f}$ homeomorphically sends each edge of $\tilde{\lambda}$ to a complete geodesic of the hyperbolic space $\mathbb{H}^{3}$, and every face of $\tilde{\lambda}$ to a totally geodesic ideal triangle of $\mathbb{H}^{3}$, with vertices on the sphere at infinity $\partial_{\infty} \mathbb{H}^{3}$;

(2) $\tilde{f}$ is $\bar{r}$-equivariant, in the sense that $\tilde{f}(\gamma \widetilde{x})=\bar{r}(\gamma)(\widetilde{f}(\widetilde{x}))$ for every $\gamma \in \pi_{1}(S)$ and every $\widetilde{x} \in \widetilde{S}$.

Following the terminology introduced in [Th], we say that the group homomorphism $\bar{r}$ : $\pi_{1}(S) \rightarrow \mathrm{PSL}_{2}(\mathbb{C})$ realizes the ideal triangulation $\lambda$ if there exists a pleated surface $(\widetilde{f}, \bar{r})$ with pleating locus $\lambda$. By extension, a point in the character variety $\mathcal{R}_{\mathrm{PSL}_{2}(\mathbb{C})}(S)$ realizes $\lambda$ if it can be represented by a homomorphism $\bar{r}: \pi_{1}(S) \rightarrow \mathrm{PSL}_{2}(\mathbb{C})$ realizing $\lambda$. Finally, a character in $\mathcal{R}_{\mathrm{SL}_{2}(\mathbb{C})}(S)$ realizes $\lambda$ if it is sent to a point of $\mathcal{R}_{\mathrm{PSL}_{2}(\mathbb{C})}(S)$ realizing $\lambda$ by the canonical projection $\mathcal{R}_{\mathrm{SL}_{2}(\mathbb{C})}(S) \rightarrow \mathcal{R}_{\mathrm{PSL}_{2}(\mathbb{C})}(S)$

We are now ready to state the result promised. At the beginning of this section, we associated a character $r_{\zeta} \in \mathcal{R}_{\mathrm{SL}_{2}(\mathbb{C})}(S)$ to each twisted homomorphism $\zeta: \mathcal{W}\left(\tau_{\lambda} ; \mathbb{Z}\right) \rightarrow \mathbb{C}^{*}$.

Proposition 16. A character $r \in \mathcal{R}_{\mathrm{SL}_{2}(\mathbb{C})}(S)$ is associated to a twisted homomorphism $\zeta: \mathcal{W}\left(\tau_{\lambda} ; \mathbb{Z}\right) \rightarrow \mathbb{C}^{*}$ as above if and only it realizes the ideal triangulation $\lambda$.

Proof. Suppose that $r \in \mathcal{R}_{\mathrm{SL}_{2}(\mathbb{C})}(S)$ realizes the ideal triangulation $\lambda$. By definition, there exists a pleated surface $(\widetilde{f}, \bar{r})$ with pleating locus $\lambda$, where the homomorphism $\bar{r}: \pi_{1}(S) \rightarrow$ $\mathrm{PSL}_{2}(\mathbb{C})$ represents the image of $r$ under the projection $\mathcal{R}_{\mathrm{SL}_{2}(\mathbb{C})}(S) \rightarrow \mathcal{R}_{\mathrm{PSL}_{2}(\mathbb{C})}(S)$.

The pleated surface $(\widetilde{f}, \bar{r})$ determines, for each edge $\widetilde{e}_{i}$ of the ideal triangulation $\widetilde{\lambda}$ of $\widetilde{S}$, a complex weight $\widetilde{x}_{i} \in \mathbb{C}^{*}$ defined as follows: If $\widetilde{Q}_{i} \subset \widetilde{S}$ is the quadrilateral formed by the two faces of $\widetilde{\lambda}$ meeting along the edge $\widetilde{e}_{i}$, then $-\widetilde{x}_{i}$ is the cross-ratio of the 4 vertices of $\widetilde{f}\left(\widetilde{Q}_{i}\right)$ in the sphere at infinity $\mathbb{C} \cup\{\infty\}$ of $\mathbb{H}^{3}$. These edge weights $\widetilde{x}_{i}$ are equivariant under the action of $\pi_{1}(S)$, and therefore descend to a system of weights $x_{i}$ for the edges $e_{i}$ of $\lambda$. The edge weights $x_{i} \in \mathbb{C}^{*}$ are the shear-bend parameters of the pleated surface $(\widetilde{f}, \bar{r})$.

Choose square roots $z_{i}=\sqrt{x_{i}}$. Then, for every closed curve $K$ in $S$, there is an explicit formula that expresses the trace $\operatorname{Tr} \bar{r}(K)$ as a Laurent polynomial in the $z_{i}$; see for instance $\left.\mathrm{BoW}_{1}, \S \S 1.3-1.4\right]$. Note that there necessarily is a sign ambiguity in this formula, as the trace of an element of $\mathrm{PSL}_{2}(\mathbb{C})$ is only defined up to sign. Another sign ambiguity occurs in the choice of the square roots $z_{i}=\sqrt{x_{i}}$.

We will use these edge weights $z_{i} \in \mathbb{C}^{*}$ to construct representations of $z^{\iota}(\lambda)$ and $\mathcal{S}^{-1}(S)$. Recall that a twisted homomorphism $\zeta: \mathcal{W}\left(\tau_{\lambda} ; \mathbb{Z}\right) \rightarrow \mathbb{C}^{*}$ is equivalent to the data of its value 
on a set of generators of $\mathcal{W}\left(\tau_{\lambda} ; \mathbb{Z}\right) \cong Z^{n}$. We can therefore find such a twisted homomorphism so that

$$
\zeta(\alpha)= \pm z_{1}^{\alpha_{1}} z_{2}^{\alpha_{2}} \ldots z_{n}^{\alpha_{n}}
$$

for every edge weight system $\alpha \in \mathcal{W}\left(\tau_{\lambda} ; \mathbb{Z}\right)$ assigning weight $\alpha_{i} \in \mathbb{Z}$ to the edge $e_{i}$ of $\lambda$. The \pm signs are here required by the twisting. In addition, a simple manipulation of the formula for the Thurston intersection form (or a use of Lemma 10) show that $\Omega(\alpha, \beta)$ is even whenever $\alpha \in(2 \mathbb{Z})^{n} \subset \mathcal{W}\left(\tau_{\lambda} ; \mathbb{Z}\right)$ assigns even weights $\alpha_{i} \in \mathbb{Z}$ to all edges of $\lambda$; in particular, there is no twisting on $(2 \mathbb{Z})^{n} \subset \mathcal{W}\left(\tau_{\lambda} ; \mathbb{Z}\right)$. Using Lemma 9 , we can therefore arrange that

$$
\zeta(\alpha)=+z_{1}^{\alpha_{1}} z_{2}^{\alpha_{2}} \ldots z_{n}^{\alpha_{n}}
$$

for every $\alpha \in(2 \mathbb{Z})^{n}$.

Note that there are several possible choices for $\zeta$, coming from the signs \pm . In fact, Lemma 9 shows that there are exactly $2^{d}$ possibilities for $\zeta$, where $d$ is the dimension of $H_{1}\left(S ; \mathbb{Z}_{2}\right)$. We will later adjust the choice of $\zeta$ so that it fits our purposes.

Let $\mu_{\zeta}: Z^{\iota}(\lambda) \rightarrow \operatorname{End}(\mathbb{C})=\mathbb{C}$ be the representation of $z^{\iota}(\lambda)$ associated by Proposition 15 to the twisted homomorphism $\zeta$. Namely, $\mu\left(Z_{\alpha}\right)=\zeta(\alpha)$ for every $\alpha \in \mathcal{W}\left(\tau_{\lambda} ; \mathbb{Z}\right)$

The definition of the quantum trace homomorphism $\operatorname{Tr}_{\lambda}^{\iota}: \mathcal{S}^{-1}(S) \rightarrow Z^{\iota}(\lambda)$ in $\mathrm{BoW}_{1}$ was specially designed to copy the formula expressing the trace $\operatorname{Tr} \bar{r}(K)$ as a Laurent polynomial in the square roots $z_{i}=\sqrt{x_{i}}$ of the shear-bend parameters of the pleated surface $(\widetilde{f}, \bar{r})$. In particular, because of the key property that $\mu_{\zeta}\left(Z_{i}^{2}\right)=+z_{i}^{2}$,

$$
\mu_{\zeta} \circ \operatorname{Tr}_{\lambda}^{\iota}([K])= \pm \operatorname{Tr} r(K)
$$

for every framed knot $K \subset S \times[0,1]$, where the sign \pm depends on $K$ and on the choice of the square roots $z_{i}=\sqrt{x_{i}}$; see the discussion in $\left[\mathrm{BoW}_{1}, \S \S 1.3-1.4\right]$.

As discussed at the beginning of this section, the homomorphism $\mu_{\zeta} \circ \operatorname{Tr}_{\lambda}^{\iota}: \mathcal{S}^{-1}(S) \rightarrow \mathbb{C}$ also defines a character $r_{\zeta} \in \mathcal{R}_{\mathrm{SL}_{2}(\mathbb{C})}(S)$ such that

$$
\mu_{\zeta} \circ \operatorname{Tr}_{\lambda}^{\iota}([K])=-\operatorname{Tr} r_{\zeta}(K)
$$

for every framed knot $K \subset S \times[0,1]$. As a consequence, $\operatorname{Tr} r_{\zeta}(K)= \pm \operatorname{Tr} r(K)$ for every knot $K$.

At this point, there is no reason for the two characters $r$ and $r_{\zeta} \in \mathcal{R}_{\mathrm{SL}_{2}(\mathbb{C})}(S)$ to coincide. However, by construction, they project to the same $\mathrm{PSL}_{2}(\mathbb{C})$-valued character in $\mathcal{R}_{\mathrm{PSL}_{2}(\mathbb{C})}(S)$. Their difference can therefore be encoded by a cohomology class $\varepsilon \in H^{1}\left(S ; \mathbb{Z}_{2}\right)$, such that

$$
\operatorname{Tr} r(K)=(-1)^{\varepsilon(K)} \operatorname{Tr} r_{\zeta}(K)
$$

for every knot $K \subset S \times[0,1]$.

Each edge $e_{i}$ of the ideal triangulation $\lambda$ is Poincaré dual to a cohomology class $\varepsilon_{i} \in$ $H^{1}\left(S ; \mathbb{Z}_{2}\right)$. Replacing the square root $z_{i}=\sqrt{x_{i}}$ by the other square root $-z_{i}$ has the effect of replacing $r_{\zeta}$ with $\varepsilon_{i} r_{\zeta}$. Since the $\varepsilon_{i}$ generate $H^{1}\left(S ; \mathbb{Z}_{2}\right)$, we can therefore adjust the choice of the square roots $z_{i}=\sqrt{x_{i}}$ so that the characters $r$ and $r_{\zeta} \in \mathcal{R}_{\mathrm{SL}_{2}(\mathbb{C})}(S)$ are now equal.

This proves that, if the character $r \in \mathcal{R}_{\mathrm{SL}_{2}(\mathbb{C})}(S)$ realizes the ideal triangulation $\lambda$, there exists a twisted homomorphism $\zeta: \mathcal{W}\left(\tau_{\lambda} ; \mathbb{Z}\right) \rightarrow \mathbb{C}^{*}$ whose associated character $r_{\zeta} \in \mathcal{R}_{\mathrm{SL}_{2}(\mathbb{C})}(S)$ is equal to $r$.

Conversely, suppose that $r=r_{\zeta} \in \mathcal{R}_{\mathrm{SL}_{2}(\mathbb{C})}(S)$ is associated to a twisted homomorphism $\zeta: \mathcal{W}\left(\tau_{\lambda} ; \mathbb{Z}\right) \rightarrow \mathbb{C}^{*}$ as above. More precisely, consider the corresponding representation $\mu_{\zeta}$ : 
$Z^{\iota}(\lambda) \rightarrow \operatorname{End}(\mathbb{C})=\mathbb{C}$, defined by the property that $\mu_{\zeta}\left(Z_{\alpha}\right)=\zeta(\alpha)$ for every $\alpha \in \mathcal{W}\left(\tau_{\lambda} ; \mathbb{Z}\right)$ Then

$$
\mu_{\zeta} \circ \operatorname{Tr}_{\lambda}^{\iota}([K])=-\operatorname{Tr} r(K)
$$

for every framed knot $K \subset S \times[0,1]$

The generator $Z_{i} \in \mathcal{T}^{\iota}(\lambda)$ associated to the edge $e_{i}$ of $\lambda$ does not satisfy the exponent parity condition defining the balanced Chekhov-Fock algebra $Z^{\iota}(\lambda)$, but its square does. We can therefore consider $x_{i}=\mu_{\zeta}\left(Z_{i}^{2}\right) \in \mathbb{C}$, which is different from 0 since $Z_{i}^{2}$ is invertible.

We can then construct a pleated surface $(\tilde{f}, \bar{r})$ whose pleating locus is equal to $\lambda$ and whose shear-bend parameters are equal to the edge weights $x_{i}$. In particular, this pleated surface is equivariant with respect to a homomorphism $\bar{r}: \pi_{1}(S) \rightarrow \mathrm{PSL}_{2}(\mathbb{C})$, which defines a character $\bar{r} \in \mathcal{R}_{\mathrm{PSL}_{2}(\mathbb{C})}(S)$.

By our discussion of the geometric interpretation of the trace homomorphism $\operatorname{Tr}_{\lambda}^{\iota}$, the character $\bar{r} \in \mathcal{R}_{\mathrm{PSL}_{2}(\mathbb{C})}(S)$ is the projection of $r \in \mathcal{R}_{\mathrm{SL}_{2}(\mathbb{C})}(S)$. In particular, $r$ realizes the ideal triangulation $\lambda$.

This concludes the proof of Proposition 16.

\section{Representations of the SKein ALGEBRA}

We are now ready to prove Theorem 2 .

We begin with an elementary lemma about the Chebyshev polynomials $T_{n}$. Remember that the polynomial $T_{n}$ is defined by the property that $\operatorname{Tr} M^{n}=T_{n}(\operatorname{Tr} M)$ for every $M \in$ $\mathrm{SL}_{2}(\mathbb{C})$. Applying this to a rotation matrix gives the trigonometric interpretation that $\cos n \theta=\frac{1}{2} T_{n}(2 \cos \theta)$.

\section{Lemma 17.}

(1) If $x=a+a^{-1}$, then $T_{n}(x)=a^{n}+a^{-n}$;

(2) If $y=b+b^{-1}$, the set of solutions to the equation $T_{n}(x)=y$ consists of the numbers $x=a+a^{-1}$ as a ranges over all $n$-roots of $b$.

Proof. For a matrix $M \in \mathrm{SL}_{2}(\mathbb{C})$, the data of its trace $x$ is equivalent to the data of its spectrum $\left\{a, a^{-1}\right\}$. The first property is then a straightfoward consequence of the fact that $\operatorname{Tr} M^{n}=T_{n}(\operatorname{Tr} M)$. The second property immediately follows.

We will also need the following quantum trace computation, connecting the skein $\left[P_{k}\right] \in$ $\mathcal{S}^{A}(S)$ and the central element $H_{k} \in \mathcal{Z}^{\omega}(\lambda)$ that are both associated to the $k$-th puncture of $S$.

Lemma 18. For the quantum trace homomorphism $\operatorname{Tr}_{\lambda}^{\omega}: \mathcal{S}^{A}(S) \rightarrow \mathcal{Z}^{\omega}(\lambda)$,

$$
\operatorname{Tr}_{\lambda}^{\omega}\left(\left[P_{k}\right]\right)=H_{k}+H_{k}^{-1}
$$

Proof. Let $e_{i_{1}}, e_{i_{2}}, \ldots, e_{i_{u}}$ be the edges of $\lambda$ that lead to the $k$-th puncture, indexed in counterclockwise order around the puncture; in particular, the $e_{i_{j}}$ are not necessarily distinct.

The construction of $\operatorname{Tr}_{\lambda}^{\omega}\left(\left[P_{k}\right]\right)$ in $\left[\mathrm{BoW}_{1}\right.$ requires a careful control of elevations (namely $[0,1]$-coordinates) along the knot $P_{k} \subset S \times[0,1]$. Choose this knot so that it steadily goes up from $e_{i_{1}}$ to $e_{i_{u}}$, and then sharply goes down to return to its starting point in $e_{i_{1}}$. In this setup, the formula of $\mathrm{BoW}_{1}$ gives that

$$
\operatorname{Tr}_{\lambda}^{\omega}\left(\left[P_{k}\right]\right)=\omega^{-u+2} Z_{i_{1}} Z_{i_{2}} \ldots Z_{i_{u}}+\omega^{-u+2} Z_{i_{1}}^{-1} Z_{i_{2}}^{-1} \ldots Z_{i_{u}}^{-1} .
$$


This is relatively straightforward when only one end of the edge $e_{i_{1}}$ leads to the $k$-th puncture, namely when the projection of $P_{k}$ to $S$ crosses $e_{i_{1}}$ only once, but otherwise requires the consideration corrections term in a bigon neighborhood of $e_{i_{1}}$, of the type given by $\mathrm{BoW}_{1}$, Lemma 22]. Fortunately, these correction terms turn out to be trivial in this case.

We need to connect this formula to $H_{k}=\left[Z_{i_{1}} Z_{i_{2}} \ldots Z_{i_{u}}\right]$. Computing the Weyl quantum ordering is again straightforward when each edge $e_{i_{j}}$ has only one end leading to the $k^{-}$ th puncture. For the general case, we could use a brute force computation as in $\mathrm{BoW}_{4}$, Lemma 12]. We prefer to give here a more indirect argument, based on the invariance of $\operatorname{Tr}_{\lambda}^{\omega}\left(\left[P_{k}\right]\right)$ under isotopy of $P_{k}$.

For this, choose the elevation of $P_{k}$ so that it now goes down from $e_{i_{1}}$ to $e_{i_{u}}$, and then goes up near $e_{i_{1}}$ to return to its starting point. The formulas of $\mathrm{BoW}_{1}$ give in this setup

$$
\operatorname{Tr}_{\lambda}^{\omega}\left(\left[P_{k}\right]\right)=\omega^{u-2} Z_{i_{u}} Z_{i_{u-1}} \ldots Z_{i_{1}}+\omega^{u-2} Z_{i_{u}}^{-1} Z_{i_{u-1}}^{-1} \ldots Z_{i_{1}}^{-1} .
$$

Comparing the two expressions for $\operatorname{Tr}_{\lambda}^{\omega}\left(\left[P_{k}\right]\right) \in z^{\omega}(\lambda)$ shows in particular that

$$
\omega^{-u+2} Z_{i_{1}} Z_{i_{2}} \ldots Z_{i_{u}}=\omega^{u-2} Z_{i_{u}} Z_{i_{u-1}} \ldots Z_{i_{1}}
$$

By definition of the Weyl quantum ordering, there exists an integer $\alpha \in \mathbb{Z}$ such that

$$
H_{k}=\omega^{\alpha} Z_{i_{1}} Z_{i_{2}} \ldots Z_{i_{u}}=\omega^{-\alpha} Z_{i_{u}} Z_{i_{u-1}} \ldots Z_{i_{1}} \text {. }
$$

We can then rephrase the above equality as $\omega^{-\alpha-u+2} H_{k}=\omega^{\alpha+u-2} H_{k}$. Although the current article usually focuses on the case where $\omega$ is a root of unity, these computations are valid for all $\omega$. It follows that $\alpha=-u+2$. This proves that $H_{k}=\omega^{-u+2} Z_{i_{1}} Z_{i_{2}} \ldots Z_{i_{u}}$, and our first computation then shows that $\operatorname{Tr}_{\lambda}^{\omega}\left(\left[P_{k}\right]\right)=H_{k}+H_{k}^{-1}$.

We are now ready to prove Theorem 2, which we repeat here for convenience. Recall that a character $r \in \mathcal{R}_{\mathrm{SL}_{2}(\mathbb{C})}(S)$ associates a number $\operatorname{Tr} r\left(P_{k}\right)$ to the $k$-th puncture of $S$, where $P_{k}$ is a small loop going around the puncture.

Theorem 19. Assume that the surface $S$ has at least one puncture, that its Euler characteristic is negative, that $A$ is a primitive $N$-root of -1 with $N$ odd, and that we are given:

(1) a character $r \in \mathcal{R}_{\mathrm{SL}_{2}(\mathbb{C})}(S)$ realizing some ideal triangulation of $S$;

(2) a number $p_{k} \in \mathbb{C}$ such that $T_{N}\left(p_{k}\right)=-\operatorname{Tr} r\left(P_{k}\right)$ for each of the punctures of $S$.

Then, there exists an irreducible finite-dimensional representation $\rho: \mathcal{S}^{A}(S) \rightarrow \operatorname{End}(E)$ whose classical shadow is equal to $r$ and whose puncture invariants are the $p_{k}$.

Proof. Since $r \in \mathcal{R}_{\mathrm{SL}_{2}(\mathbb{C})}(S)$ realizes the ideal triangulation $\lambda$, Proposition 16 provides a twisted homomorphism $\zeta: \mathcal{W}\left(\tau_{\lambda} ; \mathbb{Z}\right) \rightarrow \mathbb{C}^{*}$ and an associated representation $\mu_{\zeta}: Z^{\iota}(\lambda) \rightarrow$ $\operatorname{End}(\mathbb{C})=\mathbb{C}$, such that

$$
\mu_{\zeta} \circ \operatorname{Tr}_{\lambda}^{\iota}([K])=-\operatorname{Tr} r(K)
$$

for every framed knot $K \subset S \times[0,1]$

By Lemma 18, the image of $\left[P_{k}\right] \in \mathcal{S}^{A}(S)$ under the quantum trace homomorphism $\operatorname{Tr}_{\lambda}^{\omega}$ : $\mathcal{S}^{A}(S) \rightarrow Z^{\omega}(\lambda)$ is equal to $\operatorname{Tr}_{\lambda}^{\omega}\left(\left[P_{k}\right]\right)=H_{k}+H_{k}^{-1}$ in $z^{\omega}(\lambda)$. Similarly, $\operatorname{Tr}_{\lambda}^{\iota}\left(\left[P_{k}\right]\right)=H_{k}+H_{k}^{-1}$ in $Z^{\iota}(\lambda)$. (Beware that we are using the same symbols to denote the skeins $\left[P_{k}\right] \in \mathcal{S}^{A}(S)$ and $\left[P_{k}\right] \in \mathcal{S}^{-1}(S)$, and the central elements $H_{k} \in \mathcal{Z}^{\omega}(\lambda)$ and $H_{k} \in Z^{\iota}(\lambda)$.) Then, for $\left[P_{k}\right] \in \mathcal{S}^{-1}(S)$,

$$
\operatorname{Tr} r\left(P_{k}\right)=-\mu_{\zeta} \circ \operatorname{Tr}_{\lambda}^{\iota}\left(\left[P_{k}\right]\right)=-\mu_{\zeta}\left(H_{k}+H_{k}^{-1}\right)=-g_{k}-g_{k}^{-1}
$$

if we set $g_{k}=\mu_{\zeta}\left(H_{k}\right) \in \operatorname{End}(\mathbb{C})=\mathbb{C}$. 
For each $k$, we are given a number $p_{k} \in \mathbb{C}$ such that $T_{N}\left(p_{k}\right)=-\operatorname{Tr} r\left(P_{k}\right)=g_{k}+g_{k}^{-1}$. Lemma 17 then provides an $N$-root $h_{k}=\sqrt[N]{g_{k}}$ of such that $p_{k}=h_{k}+h_{k}^{-1}$.

Proposition 15 associates to the homomorphism $\zeta: \mathcal{W}\left(\tau_{\lambda} ; \mathbb{Z}\right) \rightarrow \mathbb{C}^{*}$ and to the $N$-roots $h_{k}=\mu_{\zeta}\left(H_{k}\right)^{\frac{1}{N}}$ an irreducible representation $\mu: Z^{\omega}(\lambda) \rightarrow \operatorname{End}(E)$ such that

(1) $\mu\left(Z_{\alpha}^{N}\right)=\zeta(\alpha) \operatorname{Id}_{E}$ for every $\alpha \in \mathcal{W}\left(\tau_{\lambda} ; \mathbb{Z}\right)$;

(2) $\mu\left(H_{k}\right)=h_{k} \operatorname{Id}_{E}$ for every $k=1, \ldots, s$.

Composing with the quantum trace map $\operatorname{Tr}_{\lambda}^{\omega}: \mathcal{S}^{A}(S) \rightarrow Z^{\omega}(\lambda)$, we now have a representation

$$
\rho=\mu \circ \operatorname{Tr}_{\lambda}^{\omega}: \mathcal{S}^{A}(S) \rightarrow \operatorname{End}(E) .
$$

Let $K$ be a framed knot whose projection to $S$ has no crossing and whose framing is vertical. Then, for the associated skein $[K] \in \mathcal{S}^{A}(S)$,

$$
T_{N}(\rho([K]))=\rho\left(T_{N}([K])\right)=\mu \circ \operatorname{Tr}_{\lambda}^{\omega}\left(T_{N}([K])\right)=\mu \circ \operatorname{Tr}_{\lambda}^{\omega} \circ \mathbf{T}^{A}([K])=\mu \circ \mathbf{F}^{\omega} \circ \operatorname{Tr}_{\lambda}^{\iota}([K])
$$

by using the fact that $\rho$ is an algebra homomorphism for the first equality, by definition of the Chebyshev homomorphism $\mathbf{T}^{A}: \mathcal{S}^{-1}(S) \rightarrow \mathcal{S}^{A}(S)$ in $\$ 1.3$ for the third equality, and by the miraculous cancellations of Theorem 7 for the last relation. In terms of the Frobenius homomorphism $\mathbf{F}^{\omega}: \mathcal{T}^{\iota}(\lambda) \rightarrow \mathcal{T}^{\omega}(\lambda)$ introduced in $\$ 1.3$ and of the representation $\mu_{\zeta}: Z^{\iota}(\lambda) \rightarrow$ $\operatorname{End}(\mathbb{C})=\mathbb{C}$, the property that $\mu\left(Z_{\alpha}^{N}\right)=\zeta(\alpha) \operatorname{Id}_{E}=\mu_{\zeta}\left(Z_{\alpha}\right)$ for every $\alpha \in \mathcal{W}\left(\tau_{\lambda} ; \mathbb{Z}\right)$ can be rephrased as $\mu \circ \mathbf{F}^{\omega}=\mu_{\zeta}$. Therefore,

$$
T_{N}(\rho([K]))=\mu \circ \mathbf{F}^{\omega} \circ \operatorname{Tr}_{\lambda}^{\iota}([K])=\mu_{\zeta} \circ \operatorname{Tr}_{\lambda}^{\iota}([K]) \operatorname{Id}_{E}=-\operatorname{Tr} r(K) \operatorname{Id}_{E} .
$$

Also, for the $k$-th puncture of $S$,

$$
\rho\left(\left[P_{k}\right]\right)=\mu \circ \operatorname{Tr}_{\lambda}^{\omega}\left(\left[P_{k}\right]\right)=\mu\left(H_{k}+H_{k}^{-1}\right)=\left(h_{k}+h_{k}^{-1}\right) \operatorname{Id}_{E}=p_{k} \operatorname{Id}_{E} .
$$

If we knew that $\rho$ was irreducible, we would be done with the proof of Theorem 19. At this point, there is no reason for this property to hold. However, if $\rho$ is not irreducible, it suffices to consider an irreducible component $\rho^{\prime}: \mathcal{S}^{A}(S) \rightarrow \operatorname{End}(F)$ with $F \subset E$. Restricting the above computations to $F$ shows that the classical shadow of the representation $\rho$ is equal to the character $r \in \mathcal{R}_{\mathrm{SL}_{2}(\mathbb{C})}(S)$, and that its puncture invariants are equal to the numbers $p_{k}$.

Remark 20. We conjecture that, when $r$ is sufficiently generic in $\mathcal{R}_{\mathrm{SL}_{2}(\mathbb{C})}(S)$, the representation $\rho=\mu \circ \operatorname{Tr}_{\lambda}^{\omega}$ used in the proof of Theorem 19 is already irreducible, and that there is no need to restrict to an irreducible factor. We also conjecture that, for generic $r \in \mathcal{R}_{\mathrm{SL}_{2}(\mathbb{C})}(S)$, there is a unique representation $\rho$ satisfying the conclusions of Theorem [19, up to isomorphism. This second conjecture was recently proved by Nurdin Takenov [Ta] for the onepuncture torus and the four-puncture sphere (building on earlier work of Bullock-Przytycki [BuP and Havlíček-Pošta [HaP] for the one-puncture torus).

Remark 21. In the very non-generic case there $r\left(P_{k}\right)$ is the identity and $p_{k}=-\omega^{4}-\omega^{-4}$ for some punctures, the representation $\rho=\mu \circ \operatorname{Tr}_{\lambda}^{\omega}$ is definitely reducible. This is a key ingredient of the "puncture filling" process developed in $\mathrm{BoW}_{4}$.

\section{A UNIQUENESS PROPERTY}

We made choices in the proof of Theorem 19. The goal of this section is to show that its output does not depend on these choices, provided we carefully specify our data and our construction. The resulting uniqueness statement will be used in the subsequent article 
$\mathrm{BoW}_{4}$, where we heavily rely on Theorem 19 and apply this statement to suitable punctured surfaces in order to construct representations of the skein algebra of a closed surface.

5.1. Pleated surfaces and representations of $z^{\iota}(\lambda)$. The proof of Theorem 19 hinges on Proposition 16 which, given a character $r \in \mathcal{R}_{\mathrm{SL}_{2}(\mathbb{C})}(S)$, provides a twisted homomorphism $\zeta: \mathcal{W}\left(\tau_{\lambda} ; \mathbb{Z}\right) \rightarrow \mathbb{C}^{*}$ and its associated representation $\mu_{\zeta}: Z^{\iota}(\lambda) \rightarrow \mathbb{C}$ such that

$$
\mu_{\zeta} \circ \operatorname{Tr}_{\lambda}^{\iota}([K])=-\operatorname{Tr} r(K)
$$

for every framed knot $K \subset S \times[0,1]$. Recall that $\mu_{\zeta}$ and $\zeta$ are related by the property that $\mu_{\zeta}\left(Z_{\alpha}\right)=\zeta(\alpha) \in \mathbb{C}^{*}$ for every basis element $Z_{\alpha} \in Z^{\iota}(\lambda)$ associated to an edge weight system $\alpha \in \mathcal{W}\left(\tau_{\lambda} ; \mathbb{Z}\right)$.

For most characters $r \in \mathcal{R}_{\mathrm{SL}_{2}(\mathbb{C})}(S)$, the homomorphism $\mu_{\zeta}: z^{\iota}(\lambda) \rightarrow \mathbb{C}$ is uniquely determined by $r$ and by the pleated surface $(\tilde{f}, \bar{r})$. However this uniqueness fails, in a very specific way, when the character $r \in \mathcal{R}_{\mathrm{SL}_{2}(\mathbb{C})}(S)$ admits a very special type of internal symmetry which we now describe.

The cohomology group $H^{1}\left(S ; \mathbb{Z}_{2}\right)$ acts on the character variety $\mathcal{R}_{\mathrm{SL}_{2}(\mathbb{C})}(S)$ by the property that, for every homomorphism $r: \pi_{1}(S) \rightarrow \mathrm{SL}_{2}(\mathbb{C})$ and cohomology class $\varepsilon \in H^{1}\left(S ; \mathbb{Z}_{2}\right)$, the homomorphism $\varepsilon r$ is defined by

$$
\varepsilon r(\gamma)=(-1)^{\varepsilon(\gamma)} r(\gamma) \in \mathrm{SL}_{2}(\mathbb{C})
$$

for every $\gamma \in \pi_{1}(S)$. We say that $\varepsilon \in H^{1}\left(S ; \mathbb{Z}_{2}\right)$ is a sign-reversal symmetry for the character $r \in \mathcal{R}_{\mathrm{SL}_{2}(\mathbb{C})}(S)$ if the action of $\varepsilon$ on $\mathcal{R}_{\mathrm{SL}_{2}(\mathbb{C})}(S)$ fixes $r$. This is equivalent to the property that the trace $\operatorname{Tr} r(\gamma)$ is equal to 0 for every $\gamma \in \pi_{1}(S)$ with $\varepsilon(\gamma) \neq 0$.

The group $H^{1}\left(S ; \mathbb{Z}_{2}\right)$ also acts on the balanced Chekhov-Fock algebra $z^{\omega}(\lambda)$ by the property that $\varepsilon Z_{\alpha}=(-1)^{\varepsilon([\alpha])} Z_{\alpha}$ for every $\varepsilon \in H^{1}\left(S ; \mathbb{Z}_{2}\right)$ and every $\alpha \in \mathcal{W}\left(\tau_{\lambda} ; \mathbb{Z}\right)$, where $[\alpha] \in H_{1}\left(S ; \mathbb{Z}_{2}\right)$ is the homology class associated to the edge weight system $\alpha$ as in Lemma 9 ,

Proposition 22. Let the pleated surface $(\widetilde{f}, \bar{r})$ have pleating locus the ideal triangulation $\lambda$, and let $r \in \mathcal{R}_{\mathrm{SL}_{2}(\mathbb{C})}(S)$ be represented by a homomorphism $r: \pi_{1}(S) \rightarrow \mathrm{SL}_{2}(\mathbb{C})$ lifting the monodromy $r: \pi_{1}(S) \rightarrow \mathrm{SL}_{2}(\mathbb{C})$ of $(\tilde{f}, \bar{r})$. Then there exists a homomorphism $\mu_{\zeta}: Z^{\iota}(\lambda) \rightarrow \mathbb{C}$, associated to a twisted homomorphism $\zeta: \mathcal{W}\left(\tau_{\lambda} ; \mathbb{Z}\right) \rightarrow \mathbb{C}^{*}$, such that

(1) for each edge $e_{i}$ of $\lambda, \mu_{\zeta}\left(Z_{i}^{2}\right)$ is equal to the shear-bend parameter $x_{i} \in \mathbb{C}^{*}$ of $e_{i}$ in the pleated surface $(\widetilde{f}, \bar{r})$;

(2) $\mu_{\zeta} \circ \operatorname{Tr}_{\lambda}^{\iota}([K])=-\operatorname{Tr} r(K)$ for every framed knot $K \subset S \times[0,1]$.

In addition, $\mu_{\zeta}$ is unique up to the action on $Z^{\iota}(\lambda)$ of an orientation-reversal symmetry $\varepsilon \in H^{1}\left(S ; \mathbb{Z}_{2}\right)$ of the character $r \in \mathcal{R}_{\mathrm{SL}_{2}(\mathbb{C})}(S)$.

We say that a homomorphism $\mu_{\zeta}: Z^{\iota}(\lambda) \rightarrow \mathbb{C}$ satisfying the above conclusions is compatible with the pleated surface $(\widetilde{f}, \bar{r})$ and the character $r \in \mathcal{R}_{\mathrm{SL}_{2}(\mathbb{C})}(S)$.

Proof of Proposition [2. The existence is provided by Proposition 16, or more precisely by its proof to guarantee that $\mu_{\zeta}\left(Z_{i}^{2}\right)=x_{i}$ for every edge $e_{i}$ of $\lambda$.

To prove the uniqueness, suppose that we are given another homomorphism $\mu_{\zeta^{\prime}}: Z^{\iota}(\lambda) \rightarrow$ $\mathbb{C}$ satisfying the same conclusions, and associated to a twisted homomorphism $\zeta^{\prime}: \mathcal{W}\left(\tau_{\lambda} ; \mathbb{Z}\right) \rightarrow$ $\mathbb{C}^{*}$. From the property that $\mu_{\zeta}\left(Z_{i}^{2}\right)=\mu_{\zeta^{\prime}}\left(Z_{i}^{2}\right)=x_{i}$, we conclude that $\mu_{\zeta}\left(Z_{\alpha}\right)^{2}=\mu_{\zeta^{\prime}}\left(Z_{\alpha}\right)^{2}$ and therefore $\mu_{\zeta}\left(Z_{\alpha}\right)= \pm \mu_{\zeta^{\prime}}\left(Z_{\alpha}\right)$ for every $\alpha \in \mathcal{W}\left(\tau_{\lambda} ; \mathbb{Z}\right)$. Since $\mu_{\zeta}$ and $\mu_{\zeta^{\prime}}$ are both algebra homomorphisms, there consequently exists a group homomorphism $\varepsilon: \mathcal{W}\left(\tau_{\lambda} ; \mathbb{Z}\right) \rightarrow \mathbb{Z}_{2}$ such that $\mu_{\zeta}\left(Z_{\alpha}\right)=(-1)^{\varepsilon(\alpha)} \mu_{\zeta^{\prime}}\left(Z_{\alpha}\right)$ for every $\alpha \in \mathcal{W}\left(\tau_{\lambda} ; \mathbb{Z}\right)$. Another application of the property 
that $\mu_{\zeta}\left(Z_{i}^{2}\right)=\mu_{\zeta^{\prime}}\left(Z_{i}^{2}\right)$ shows that $\varepsilon$ is trivial on the subgroup $(2 \mathbb{Z})^{n} \subset \mathcal{W}\left(\tau_{\lambda} ; \mathbb{Z}\right)$ of Lemma 9 , This statement then shows that $\varepsilon$ comes from a homomorphism $H_{1}\left(S ; \mathbb{Z}_{2}\right) \rightarrow \mathbb{Z}_{2}$, and can therefore be interpreted as a cohomology class $\varepsilon \in H^{1}\left(S ; \mathbb{Z}_{2}\right)$.

In this cohomological interpretation of $\varepsilon \in H^{1}\left(S ; \mathbb{Z}_{2}\right)$, we have that $\mu_{\zeta}\left(Z_{\alpha}\right)=(-1)^{\varepsilon([\alpha])} \mu_{\zeta^{\prime}}\left(Z_{\alpha}\right)$ for every $\alpha \in \mathcal{W}\left(\tau_{\lambda} ; \mathbb{Z}\right)$. Namely, the homomorphisms $\mu_{\zeta}, \mu_{\zeta^{\prime}}: Z^{\iota}(\lambda) \rightarrow \mathbb{C}$ differ by the action of $\varepsilon \in H^{1}\left(S ; \mathbb{Z}_{2}\right)$ on $Z^{\iota}(\lambda)$.

Given a framed link $K \subset S \times[0,1]$, the construction of the quantum trace $\operatorname{Tr}_{\lambda}^{\iota}$ in $\mathrm{BoW}_{1}$ shows that $\operatorname{Tr}_{\lambda}^{\iota}([K]) \in Z^{\iota}(\lambda)$ is a linear combination of monomials $Z_{\alpha}$ whose associated homology class $[\alpha] \in H_{1}\left(S ; \mathbb{Z}_{2}\right)$, in the sense of Lemma 9 , is the same as the class $[K] \in$ $H_{1}\left(S ; \mathbb{Z}_{2}\right)$ defined by $K$. As a consequence,

$$
\operatorname{Tr} r(K)=-\mu_{\zeta^{\prime}} \circ \operatorname{Tr}_{\lambda}^{\iota}([K])=-(-1)^{\varepsilon(K)} \mu_{\zeta} \circ \operatorname{Tr}_{\lambda}^{\iota}([K])=(-1)^{\varepsilon(K)} \operatorname{Tr} r(K)
$$

for every framed link $K \subset S \times[0,1]$. This proves that $\varepsilon \in H^{1}\left(S ; \mathbb{Z}_{2}\right)$ is a sign-reversal symmetry for the character $r \in \mathcal{R}_{\mathrm{SL}_{2}(\mathbb{C})}(S)$.

As a consequence, the homomorphisms $\mu_{\zeta}, \mu_{\zeta^{\prime}}: z^{\iota}(\lambda) \rightarrow \mathbb{C}$ differ by the action on $z^{\iota}(\lambda)$ of a sign-reversal symmetry $\varepsilon \in H^{1}\left(S ; \mathbb{Z}_{2}\right)$ of $r \in \mathcal{R}_{\mathrm{SL}_{2}(\mathbb{C})}(S)$.

Characters with non-trivial sign-reversal symmetries exist, but are rare. In fact, the characters that have no (non-trivial) sign-reversal symmetries form a Zariski dense closed subset in $\mathcal{R}_{\mathrm{SL}_{2}(\mathbb{C})}(S)$. (Hint: Choose a family of simple closed curves $\gamma_{1}, \gamma_{2}, \ldots, \gamma_{k}$ in $S$ that generate $H_{1}\left(S ; \mathbb{Z}_{2}\right)$, and consider the set of $r \in \mathcal{R}_{\mathrm{SL}_{2}(\mathbb{C})}(S)$ such that $\operatorname{Tr} r\left(\gamma_{i}\right) \neq 0$ for some $i$.) This subset of $\mathcal{R}_{\mathrm{SL}_{2}(\mathbb{C})}(S)$ includes all injective homomorphisms $\pi_{1}(S) \rightarrow \mathrm{SL}_{2}(\mathbb{C})$, since their images contain no matrix with trace 0 . In particular all "geometric" characters, corresponding to fuchsian or quasifuchsian groups, admit no sign-reversal symmetries..

More precisely, a simple algebraic manipulation shows that every character $r \in \mathcal{R}_{\mathrm{SL}_{2}(\mathbb{C})}(S)$ with a non-trivial sign-reversal symmetry $\varepsilon \in H^{1}\left(S ; \mathbb{Z}_{2}\right)$ is represented by a homomorphism $r: \pi_{1}(S) \rightarrow \mathrm{SL}_{2}(\mathbb{C})$ of the following type: Considering $\varepsilon$ as a group homomorphism $\varepsilon: \pi_{1}(S) \rightarrow \mathbb{Z}_{2}$ and for an arbitrary $\gamma_{0} \in \pi_{1}(S)$ with $\varepsilon\left(\gamma_{0}\right) \neq 0$, there exists a group homomorphism $\theta: \operatorname{ker} \varepsilon \rightarrow \mathbb{C} / 2 \pi \mathrm{i} \mathbb{Z}$ such that

$$
r\left(\gamma_{0}\right)=\left(\begin{array}{cc}
\mathrm{i} & 0 \\
0 & -\mathrm{i}
\end{array}\right) \text { and } r(\gamma)=\left(\begin{array}{cc}
\cosh \theta(\gamma) & \sinh \theta(\gamma) \\
\sinh \theta(\gamma) & \cosh \theta(\gamma)
\end{array}\right)
$$

for every $\gamma \in \operatorname{ker} \varepsilon$. In particular, noting the constraints that $\theta\left(\gamma_{0}^{2}\right)=\pi \mathrm{i}$ and $\theta\left(\gamma_{0} \gamma \gamma_{0}^{-1}\right)=$ $-\theta(\gamma)$ for every $\gamma \in \operatorname{ker} \varepsilon$, the space of such characters has complex dimension $2 g+s-2$ in the $(6 g+3 s-6)$-dimensional character variety $\mathcal{R}_{\mathrm{SL}_{2}(\mathbb{C})}(S)$ (where $g$ is the genus of the surface $S$ and $s$ is its number of punctures).

5.2. A strengthening of Theorem 19. Recall that, if the $k$-th puncture of $S$ is adjacent to the edges $e_{i_{1}}, i_{2}, \ldots, e_{i_{u}}$ of the ideal triangulation $\lambda$, it determines an element $H_{k}=$ $\left[Z_{i_{1}} Z_{i_{2}} \ldots Z_{i_{u}}\right] \in Z^{\iota}(\lambda)$.

Proposition 23. Assume that the surface $S$ has at least one puncture, that its Euler characteristic is negative, that $A$ is a primitive $N$-root of -1 with $N$ odd, and that we are given:

(i) a pleated surface $(\tilde{f}, \bar{r})$ with pleating locus $\lambda$, a character $r \in \mathcal{R}_{\mathrm{SL}_{2}(\mathbb{C})}(S)$ lifting $\bar{r} \in$ $\mathcal{R}_{\mathrm{PSL}_{2}(\mathbb{C})}(S)$, and a homomorphism $\mu_{\zeta}: Z^{\iota}(\lambda) \rightarrow \mathbb{C}$ compatible with $(\widetilde{f}, \bar{r})$ and $r$ as in Proposition 22:

(ii) for each puncture of $S$ an $N$-root $h_{k}$ of $\mu_{\zeta}\left(H_{k}\right) \in \mathbb{Z}^{*}$. 
Then, up to isomorphism, there exists a unique representation $\mu: z^{\omega}(\lambda) \rightarrow \operatorname{End}(E)$ of the balanced Chekhov-Fock algebra $Z^{\omega}(\lambda)$ with the following properties:

(1) the dimension of the vector space $E$ is equal to $N^{3 g+s-3}$, where $g$ is the genus of the surface $S$ and $s$ its number of punctures;

(2) $\mu\left(Z_{\alpha}^{N}\right)=\mu_{\zeta}\left(Z_{\alpha}\right)$ for every edge weight system $\alpha \in \mathcal{W}\left(\tau_{\lambda} ; \mathbb{Z}\right)$, where we use the same symbol to represent the associated base elements $Z_{\alpha} \in Z^{\omega}(\lambda)$ and $Z_{\alpha} \in Z^{\iota}(\lambda)$;

(3) $\mu\left(H_{k}\right)=h_{k} \operatorname{Id}_{E}$ for the central element $H_{k} \in z^{\omega}(\lambda)$ associated to the $k$-th puncture of $S$.

In addition, the representation $\mu$ is irreducible and the representation $\rho=\mu \circ \operatorname{Tr}_{\lambda}^{\omega}: \mathcal{S}^{A}(S) \rightarrow$ $\operatorname{End}(E)$ has classical shadow $r \in \mathcal{R}_{\mathrm{SL}_{2}(\mathbb{C})}(S)$, in the sense that

$$
T_{N}(\rho([K]))=-\operatorname{Tr} r(K) \operatorname{Id}_{E}
$$

for every knot $K \subset S \times[0,1]$ whose projection to $S$ has no crossing and whose framing is vertical (where $T_{N}(x)$ is the $N$-th Chebyshev polynomial of the first type).

Proof. The existence and uniqueness part is essentially a restatement of the classification of irreducible representations of $Z^{\omega}(\lambda)$ in Proposition 15. The fact that $\rho$ has classical shadow $r$ follows from the proof of Theorem 19.

Although the representation $\mu: z^{\omega}(\lambda) \rightarrow \operatorname{End}(E)$ of Proposition 23 is irreducible, the representation $\rho=\mu \circ \operatorname{Tr}_{\lambda}^{\omega}: \mathcal{S}^{A}(S) \rightarrow \operatorname{End}(E)$ is not necessarily irreducible; see Remark 21 .

\section{THE CASE WHERE $A^{N}=+1$}

The case $A^{N}=+1$ can be deduced from the case $A^{N}=-1$ by the Barrett isomorphism $B_{\sigma}: \mathcal{S}^{A}(S) \rightarrow \mathcal{S}^{-A}(S)$ associated to a spin structure $\sigma$ on the surface $S$. This isomorphism is defined by the property that, for every framed link $K \subset S \times[0,1]$ with $k$ components,

$$
B_{\sigma}([K])=(-1)^{k+\sigma(K)}[K] \in \mathcal{S}^{-A}
$$

where $\sigma(K) \in \mathbb{Z}_{2}$ is the monodromy of the framing of $K$ with respect to $\sigma$. See [Ba] and [PrS, §2] for a proof that $B_{\sigma}: \mathcal{S}^{A}(S) \rightarrow \mathcal{S}^{-A}(S)$ is an algebra isomorphism.

If $A^{N}=+1$, an irreducible finite-dimensional representation $\rho: \mathcal{S}^{A}(S) \rightarrow \operatorname{End}(E)$ defines an irreducible representation $\rho^{\prime}=\rho \circ B_{\sigma}: \mathcal{S}^{-A}(S) \rightarrow \operatorname{End}(E)$, to which we can apply Theorems 1 and 2 since $(-A)^{N}=-1$ as $N$ is assumed to be odd. This process depends on the choice of a spin structure $\sigma$, but we can make if more canonical by the following construction.

Let $\operatorname{Spin}(S)$ denote the set of isotopy classes of spin structures on $S$. Any two spin structures differ by an obstruction in $H^{1}\left(S ; \mathbb{Z}_{2}\right)$, which defines an action of $H^{1}\left(S ; \mathbb{Z}_{2}\right)$ on $\operatorname{Spin}(S)$. The cohomology group $H^{1}\left(S ; \mathbb{Z}_{2}\right)$ also acts on the character variety $\mathcal{R}_{\mathrm{SL}_{2}(\mathbb{C})}(S)$ by the property that, if $r \in \mathcal{R}_{\mathrm{SL}_{2}(\mathbb{C})}(S)$ and $\alpha \in H^{1}\left(S ; \mathbb{Z}_{2}\right)$, then $\alpha r \in \mathcal{R}_{\mathrm{SL}_{2}(\mathbb{C})}(S)$ is defined by

$$
\alpha r(\gamma)=(-1)^{\alpha(\gamma)} r(\gamma) \in \mathrm{SL}_{2}(\mathbb{C})
$$

for every $\gamma \in \pi_{1}(S)$.

The twisted character variety $\mathcal{R}_{\mathrm{PSL}_{2}(\mathbb{C})}^{\mathrm{Spin}}(S)$ is then defined as the quotient

$$
\mathcal{R}_{\mathrm{PSL}_{2}(\mathbb{C})}^{\mathrm{Spin}}(S)=\left(\mathcal{R}_{\mathrm{SL}_{2}(\mathbb{C})}(S) \times \operatorname{Spin}(S)\right) / H^{1}\left(S ; \mathbb{Z}_{2}\right) .
$$

If the twisted character $\widehat{r} \in \mathcal{R}_{\mathrm{PSL}_{2}(\mathbb{C})}^{\mathrm{Spin}}(S)$ is represented by $(r, \sigma) \in \mathcal{R}_{\mathrm{SL}_{2}(\mathbb{C})}(S) \times \operatorname{Spin}(S)$ and if $K$ is a framed knot in $S \times[0,1]$, the definition is designed so that the following trace

$$
\operatorname{Tr} \widehat{r}(K)=-(-1)^{\sigma(K)} \operatorname{Tr} r(K) .
$$


depends only on the twisted character $\widehat{r} \in \mathcal{R}_{\mathrm{PSL}_{2}(\mathbb{C})}^{\mathrm{Spin}}(S)$, and not on its representative $(r, \sigma) \in$ $\mathcal{R}_{\mathrm{SL}_{2}(\mathbb{C})}(S) \times \operatorname{Spin}(S)$.

The correspondence $\rho \leftrightarrow \rho \circ B_{\sigma}$ is used in $\left[\mathrm{BoW}_{3}\right.$ to establish the following result.

Theorem $24\left(\left[\mathrm{BoW}_{3}\right)\right.$. Suppose that $A$ is a primitive $N$-root of +1 with $N$ odd, and let $\rho: \mathcal{S}^{A}(S) \rightarrow \operatorname{End}(E)$ be an irreducible finite-dimensional representation of the Kauffman bracket skein algebra. Let $T_{N}(x)$ be the $N$-th normalized Chebyshev polynomial of the first kind.

(1) There exists a unique twisted character $\widehat{r}_{\rho} \in \mathcal{R}_{\mathrm{PSL}_{2}(\mathbb{C})}^{\mathrm{Spin}_{1}}(S)$ such that

$$
T_{N}(\rho([K]))=-\left(\operatorname{Tr} \widehat{r}_{\rho}(K)\right) \operatorname{Id}_{E}
$$

for every framed knot $K \subset S \times[0,1]$ whose projection to $S$ has no crossing and whose framing is vertical.

(2) Let $P_{k}$ be a small simple loop going around the $k$-th puncture of $S$, and consider it as a knot in $S \times[0,1]$ with vertical framing. Then there exists a number $p_{k} \in \mathbb{C}$ such that $\rho\left(\left[P_{k}\right]\right)=p_{k} \operatorname{Id}_{E}$.

(3) The number $p_{k}$ of $(2)$ is related to the twisted character $\widehat{r}_{\rho} \in \mathcal{R}_{\mathrm{PSL}_{2}(\mathbb{C})}^{\mathrm{Spin}}(S)$ of $(1)$ by the property that $T_{N}\left(p_{k}\right)=-\operatorname{Tr} \widehat{r}_{\rho}\left(P_{k}\right)$.

The same correspondence $\rho \leftrightarrow \rho \circ B_{\sigma}$ can be used to prove the following analogue of Theorem 2. We say that the twisted character $\widehat{r} \in \mathcal{R}_{\mathrm{PSL}_{2}(\mathbb{C})}^{\mathrm{Spin}}(S)$ realizes the ideal triangulation $\lambda$ of $S$ if the image $\bar{r} \in \mathcal{R}_{\mathrm{PSL}_{2}(\mathbb{C})}(S)$ of $\widehat{r}$ under the natural projection $\mathcal{R}_{\mathrm{PSL}_{2}(\mathbb{C})}^{\mathrm{Spin}}(S) \rightarrow \mathcal{R}_{\mathrm{PSL}_{2}(\mathbb{C})}(S)$ realizes $\lambda$ in the sense of $\$ 3$.

Theorem 25. Assume that the surface $S$ has at least one puncture, that its Euler characteristic is negative, that $A$ is a primitive $N$-root of +1 with $N$ odd, and that we are given:

(1) a twisted character $\hat{r} \in \mathcal{R}_{\mathrm{PSL}_{2}(\mathbb{C})}^{\mathrm{Spin}}(S)$ which realizes some ideal triangulation $\lambda$ of $S$;

(2) a number $p_{k} \in \mathbb{C}$ such that $T_{N}\left(p_{k}\right)=-\operatorname{Tr} \widehat{r}\left(P_{k}\right)$ for each of the punctures of $S$.

Then, there exists an irreducible finite-dimensional representation $\rho: \mathcal{S}^{A}(S) \rightarrow \operatorname{End}(E)$ whose classical shadow is equal to $\widehat{r}$ and whose puncture invariants are the $p_{k}$.

Proof. Represent $\widehat{r} \in \mathcal{R}_{\mathrm{PSL}_{2}(\mathbb{C})}^{\mathrm{Spin}}(S)$ by a pair $(r, \sigma) \in \mathcal{R}_{\mathrm{SL}_{2}(\mathbb{C})}(S) \times \operatorname{Spin}(S)$. Theorem 2 then provides an irreducible representation $\rho^{\prime}: \mathcal{S}^{-A}(S) \rightarrow \operatorname{End}(E)$ with classical shadow $r \in \mathcal{R}_{\mathrm{SL}_{2}(\mathbb{C})}(S)$ and puncture invariants equal to the $p_{k}$. Then, $\rho=\rho^{\prime} \circ B_{\sigma}: \mathcal{S}^{A}(S) \rightarrow \operatorname{End}(E)$ satisfies the required properties.

\section{Appendix. The Thurston intersection FORM OF A TRAin TRACK}

Let $\tau$ be a train track in the surface $S$, and let $\mathcal{W}(\tau ; \mathbb{Z})$ be the space of integer valued edge weights for $\tau$. Namely, an element $\alpha \in \mathcal{W}(\tau ; \mathbb{Z})$ assigns a weight $\alpha(e) \in \mathbb{Z}$ to each edge $e$ of $\tau$ in such a way that, at each switch $s$ of $\tau$, the sum of the weight of the edges of $\tau$ coming in on one side of $s$ is equal to the sum of the weights of the edges going out on the other side. This abelian group comes with an additional structure provided by the Thurston intersection form

$$
\Omega: \mathcal{W}(\tau ; \mathbb{Z}) \times \mathcal{W}(\tau ; \mathbb{Z}) \rightarrow \mathbb{Z}
$$


defined as in \$2.2. Namely,

$$
\Omega(\alpha, \beta)=\frac{1}{2} \sum_{e \text { right of } e^{\prime}}\left(\alpha(e) \beta\left(e^{\prime}\right)-\alpha\left(e^{\prime}\right) \beta(e)\right)
$$

where the sum is over all pairs $\left(e, e^{\prime}\right)$ where $e$ and $e^{\prime}$ are two "germs of edges" emerging on the same side of a switch of $\tau$ with $e$ to the right of $e^{\prime}\left(e\right.$ and $e^{\prime}$ are not necessarily adjacent at that switch). At this point, $\Omega(\alpha, \beta)$ is only a half-integer, but Theorem [26] below will prove that it is indeed an integer.

We want to determine the algebraic structure of $\mathcal{W}(\tau ; \mathbb{Z})$ endowed with $\Omega$. This is a classical property in the case of real-valued edge weights (see for instance [PeH, §3.2] or [Bo, $\S 3]$ ), but the subtleties of the integer-valued case seem less well known. The result is of independent interest because, beyond the scope of this article, integer-valued edge weight do occur in geometric situations where the Thurston intersection form is also relevant. One such instance arises for general pleated surfaces where the pleating locus is allowed to have uncountably many leaves, as opposed to the simpler pleated surfaces considered in $\$ 3$. The bending of such a pleated surface is measured by an edge weight system valued in $\mathbb{R} / 2 \pi \mathbb{Z}$ for a train track carrying the pleating locus, and this edge weight system is related to rotation numbers by the Thurston intersection form [Bo].

The complement $S-\tau$ of the train track $\tau$ admits a certain number of "spikes", each locally delimited by two edges of $\tau$ that approach the same side of a switch of $\tau$. Thicken $\tau$ to a subsurface $U \subset S$ that deformation retracts to $\tau$. Each component of $U-\tau$ is then an annulus that contains one component of $\partial U$ and a certain number of spikes of $S-\tau$. We can then consider the genus $h$ of $U$, and the number $n_{\text {even }}$ (resp. $n_{\text {odd }}$ ) of components of $U-\tau$ that contain an even (resp. odd) number of spikes.

A component $U_{1}$ of $U-\tau$ that contains an even number $n_{1}>0$ of spikes of $S-\tau$ determines, up to sign, an element of $\mathcal{W}(\tau ; \mathbb{Z})$ as follows. The core of $U_{1}$ is homotopic to a closed curve $\gamma_{1}$ in $\tau$ that is made up of arcs $k_{1}, k_{2}, \ldots, k_{n_{1}}, k_{n_{1}+1}=k_{1}$, in this order, such that each arc $k_{1}$ is immersed in $\tau$ and such that two consecutive arcs $k_{i}$ and $k_{i+1}$ locally bound a spike of $U_{1}$ at their common end point. For each edge $e$ of $\tau$, we can then consider

$$
\alpha(e)=\sum_{i=1}^{n_{1}}(-1)^{i} \alpha_{i}(e) \in \mathbb{Z}
$$

where $\alpha_{i}(e) \in\{0,1,2\}$ is the number of times the arc $k_{i}$ passes over the edge $e$. Because the signs $(-1)^{i}$ alternate at the spikes of $U_{1}$ (using the fact that $n_{1}$ is even for $i=n_{1}$ ), one easily sees that these edge weights $\alpha(e)$ satisfy the switch conditions, and therefore define an edge weight system $\alpha \in \mathcal{W}(\tau ; \mathbb{Z})$.

A component $U_{1}$ of $U-\tau$ that contains no spike similarly determines an edge weight system $\alpha \in \mathcal{W}(\tau ; \mathbb{Z})$. The core of $U_{1}$ is now homotopic to a closed curve $\gamma_{1}$ immersed in $\tau$, and $\alpha$ associates to each edge $e$ the number $\alpha(e)$ of times $\gamma_{1}$ passes over $e$.

Theorem 26. For a connected train track $\tau$ in the surface $S$, let the numbers $h$, $n_{\text {even }}$ and $n_{\text {odd }}$ be defined as above. Then, the lattice $\mathcal{W}(\tau ; \mathbb{Z})$ of integer valued edge weight systems for $\tau$ admits a basis in which the Thurston intersection form $\Omega$ is block diagonal with

- $h$ blocks $\left(\begin{array}{cc}0 & 1 \\ -1 & 0\end{array}\right), \frac{1}{2} n_{\text {odd }}-1$ blocks $\left(\begin{array}{cc}0 & 2 \\ -2 & 0\end{array}\right)$, and $n_{\text {even }}$ blocks $(0)$ if $n_{\text {odd }}>0$;

- $h$ blocks $\left(\begin{array}{cc}0 & 1 \\ -1 & 0\end{array}\right)$ and $n_{\text {even }}$ blocks $(0)$ if $n_{\mathrm{odd}}=0$ and $\tau$ is non-orientable; 
- $h$ blocks $\left(\begin{array}{cc}0 & 1 \\ -1 & 0\end{array}\right)$ and $n_{\mathrm{even}}-1$ blocks $(0)$ if $n_{\mathrm{odd}}=0$ and $\tau$ is orientable.

In addition, in all cases, we can choose the base elements corresponding to the blocks (0) to be edge weight systems associated as above to components of $U-\tau$ that contain an even number of spikes.

In particular, $n_{\text {odd }}$ is always even.

Proof. We will subdivide the proof into several lemmas. The reader may recognize many analogies with the arguments used in the proof of [BoL, Prop. 5].

We first discuss a classical homological interpretation of the elements of $\mathcal{W}(\tau ; \mathbb{Z})$ and of the Thurston intersection form $\Omega$.

Because the edges of $\tau$ are not oriented, an edge weight system does not directly define a homology class in $H_{1}(\tau ; \mathbb{Z})$. Instead consider the 2 -fold orientation covering $\widehat{\tau}$ of $\tau$, consisting of all pairs $(x, o)$ where $x \in \tau$ and $o$ is a local orientation of the train track $\tau$ at $x$. Note that $\widehat{\tau}$ is a canonically oriented train track, and that the covering involution $\sigma: \widehat{\tau} \rightarrow \widehat{\tau}$ that exchanges the two sheets of the covering reverses the orientation of $\widehat{\tau}$.

An edge weight system $\alpha \in \mathcal{W}(\tau ; \mathbb{Z})$ lifts to a weight system $\widehat{\alpha} \in \mathcal{W}(\widehat{\tau} ; \mathbb{Z})$. Endowing each (oriented) edge of $\widehat{\tau}$ with the weight assigned by $\widehat{\alpha}$ defines a chain, which is closed because of the switch condition and therefore defines a homology class $[\widehat{\alpha}] \in H_{1}(\widehat{\tau} ; \mathbb{Z})$. Note that $\sigma_{*}([\widehat{\alpha}])=-[\widehat{\alpha}]$ since the covering involution $\sigma$ reverses the canonical orientation of $\widehat{\tau}$.

Conversely, each homology class $[\widehat{\alpha}] \in H_{1}(\widehat{\tau} ; \mathbb{Z})$ is represented by a unique linear combination of the edges of $\widehat{\tau}$, and therefore determines an edge weight system $\widehat{\alpha} \in \mathcal{W}(\widehat{\tau} ; \mathbb{Z})$. Assuming in addition that $\sigma_{*}([\widehat{\alpha}])=-[\widehat{\alpha}]$, this edge weight system is invariant under the action of $\sigma$, and therefore comes from an edge weight system $\alpha \in \mathcal{W}(\tau ; \mathbb{Z})$. This proves:

Lemma 27. The above correspondence identifies the space $\mathcal{W}(\tau ; \mathbb{Z})$ of edge weight systems to the eigenspace

$$
H_{1}(\widehat{\tau} ; \mathbb{Z})^{-}=\left\{[\widehat{\alpha}] \in H_{1}(\widehat{\tau} ; \mathbb{Z}) ; \sigma_{*}([\widehat{\alpha}])=-[\widehat{\alpha}]\right\} \subset H_{1}(\widehat{\tau} ; \mathbb{Z})
$$

of the homomorphism $\sigma_{*}: H_{1}(\widehat{\tau} ; \mathbb{Z}) \rightarrow H_{1}(\widehat{\tau} ; \mathbb{Z})$ induced by $\sigma$.

To describe the Thurston intersection form in this homological framework, consider the subsurface $U$ deformation retracting to $\tau$. The covering $\widehat{\tau} \rightarrow \tau$ uniquely extends to a 2 -fold covering $\widehat{U} \rightarrow U$, whose covering involution $\sigma: \widehat{U} \rightarrow \widehat{U}$ extends our previous involution $\sigma$.

Lemma 28. If $[\widehat{\alpha}],[\widehat{\beta}] \in H_{1}(\widehat{\tau})^{-}$are associated to the edge weight systems $\alpha, \beta \in \mathcal{W}\left(\tau_{\lambda} ; \mathbb{Z}\right)$,

$$
\Omega(\alpha, \beta)=\frac{1}{2}[\widehat{\alpha}] \cdot[\widehat{\beta}]
$$

where $\cdot$ denotes the algebraic intersection number of classes of $H_{1}(\widehat{U} ; \mathbb{Z}) \cong H_{1}(\widehat{\tau} ; \mathbb{Z})$. In addition, $[\widehat{\alpha}] \cdot[\widehat{\beta}]$ is even, and $\Omega(\alpha, \beta)$ is an integer.

Proof. To prove the first statement push the oriented train track $\widehat{\tau}$ to its left to obtain a train track $\widehat{\tau}^{\prime} \subset \widehat{U}$ that is transverse to $\widehat{\tau}$, realize the homology class $[\widehat{\alpha}]$ by $\widehat{\tau}$ endowed with the edge multiplicities coming from $\alpha$, realize $[\widehat{\beta}]$ by $\widehat{\tau}^{\prime}$ endowed with the edge multiplicities coming from $\beta$, and use this setup to compute the algebraic intersection number $[\widehat{\alpha}] \cdot[\widehat{\beta}]$. Evaluating the contribution to $[\widehat{\alpha}] \cdot[\widehat{\beta}]$ of each point of $\widehat{\tau} \cap \widehat{\tau}^{\prime}$ then shows that this algebraic intersection number is equal to $2 \Omega(\alpha, \beta)$. 
The second statement is obtained by a similar but different computation of $[\widehat{\alpha}] \cdot[\widehat{\beta}]$. Perturb $\tau$ to a train track $\tau^{\prime \prime}$ that is transverse to $\tau$, and let $\widehat{\tau}^{\prime \prime}$ be the pre-image of $\tau^{\prime \prime}$ in $\widehat{U}$. Now compute $[\widehat{\alpha}] \cdot[\widehat{\beta}]$ by realizing the homology class $[\widehat{\beta}]$ by $\widehat{\tau}^{\prime \prime}$ endowed with the edge multiplicities coming from $\beta$, while still realizing $[\widehat{\alpha}]$ by $\widehat{\tau}$ endowed with the edge multiplicities coming from $\alpha$. The intersection $\widehat{\tau} \cap \widehat{\tau}^{\prime \prime}$ splits into pairs of points interchanged by the covering involution $\sigma$, and the two points in each pair have the same contribution to $[\widehat{\alpha}] \cdot[\widehat{\beta}]$. It follows that $[\widehat{\alpha}] \cdot[\widehat{\beta}]$ is even.

We now need to better understand the action of $\sigma_{*}$ on the homology group $H_{1}(\widehat{U} ; \mathbb{Z})$.

It will be convenient to systematically use a notation which already appeared in Lemma 27. If $V$ is a space where some restriction of the covering involution $\sigma$ induces a homomorphism $\sigma_{*}$, then

$$
V^{-}=\left\{\alpha \in V ; \sigma_{*}(\alpha)=-\alpha\right\} .
$$

For instance, Lemma 27 provides a natural isomorphism $\mathcal{W}(\tau ; \mathbb{Z}) \cong H_{1}(\widehat{U} ; \mathbb{Z})^{-}$.

Let $\partial_{\text {even }} U$ be the union of the $n_{\text {even }}$ components of $\partial U$ that are adjacent to an even number of spikes to $S-\tau$, and set $\partial_{\text {odd }} U=\partial U-\partial_{\text {even }} U$.

Lemma 29. Let $\gamma_{1}$ be a component of $\partial_{\text {even }} U$, and let $\widehat{\gamma}_{1}$ be its pre-image in $\widehat{U}$. Then $\left.H_{1}\left(\widehat{\gamma}_{1}\right) ; \mathbb{Z}\right)^{-} \cong \mathbb{Z}$, and the image in $H_{1}(\widehat{U} ; \mathbb{Z})^{-} \cong \mathcal{W}(\tau ; \mathbb{Z})$ of one of its generators coincides up to sign with the edge weight system that we associated right before Theorem 26 to the component $U_{1}$ of $U-\tau$ that contains $\gamma_{1}$.

Proof. As right above Theorem [26, the curve $\gamma_{1}$ is homotopic to a closed curve $\gamma_{1}^{\prime}$ in $\tau$ that is made up of $n_{1} \operatorname{arcs} k_{1}, k_{2}, \ldots, k_{n_{1}}, k_{n_{1}+1}=k_{1}$, in this order, such that each arc $k_{1}$ is immersed in $\tau$ and such that two consecutive arcs $k_{i}$ and $k_{i+1}$ locally bound a spike of $U_{1}$ at their common end point. Because $n_{1}$ is even, there are two possible ways to orient these arcs in such a way that consecutive arcs have opposite orientations. This shows that $\gamma_{1}^{\prime}$ has two distinct lifts to $\widehat{\tau}$, and therefore that the pre-image $\widehat{\gamma}_{1}$ of $\gamma_{1}$ in $\widehat{U}$ consists of two components of $\partial \widehat{U}$ that are exchanged by the covering involution. This provides an isomorphism $H_{1}\left(\widehat{\gamma}_{1} ; \mathbb{Z}\right) \cong \mathbb{Z} \oplus \mathbb{Z}$ where $\sigma_{*}$ exchanges the two factors. It immediately follows that $H_{1}\left(\widehat{\gamma}_{1} ; \mathbb{Z}\right)^{-} \cong \mathbb{Z}$.

If $\widehat{\gamma}_{1}^{\prime} \subset \widehat{\tau}$ denotes one of the two lifts of $\gamma_{1}^{\prime}$ to $\widehat{\tau}$, the image of $H_{1}\left(\widehat{\gamma}_{1} ; \mathbb{Z}\right)^{-}$in $H_{1}(\widehat{U} ; \mathbb{Z})^{-} \cong$ $H_{1}(\widehat{\tau} ; \mathbb{Z})^{-} \cong \mathcal{W}(\tau ; \mathbb{Z})$ is generated by $\left[\widehat{\gamma}_{1}^{\prime}\right]-\sigma_{*}\left(\left[\widehat{\gamma}_{1}^{\prime}\right]\right)$. The second statement easily follows.

To prove Theorem 26, we will first restrict attention to the case where $n_{\text {odd }}>0$. This is equivalent to the property that $\partial_{\text {odd }} U$ is non-empty.

We just saw that the restriction of the covering $\widehat{U} \rightarrow U$ above $\partial_{\text {even }} U$ is trivial; similarly, its restriction above each component of $\partial_{\text {odd }} U$ is non-trivial. Therefore, the covering $\widehat{U} \rightarrow U$ is classified by a cohomology class in $H^{1}\left(U ; \mathbb{Z}_{2}\right)$ which evaluates to 0 on the elements of $\partial_{\text {even }} U$ and to 1 on the components of $\partial_{\text {odd }} U$.

Since the subset $\partial_{\text {odd }} U$ is non-empty, and can therefore realize the cohomology class classifying the covering $\widehat{U} \rightarrow U$ as the Poincaré dual of a family $K \subset U$ of disjoint arcs whose boundary $\partial K=K \cap \partial U$ consists of one point in each component of $\partial_{\text {odd }} U$.

Split $U$ along a separating simple closed curve $\gamma$ to isolate $K$ inside of a planar surface $U_{1} \subset S$ with boundary $\partial U_{1}=\gamma \cup \partial_{\text {odd }} U$, while the closure $U_{2}$ of $U-U_{1}$ has genus $h$ and boundary $\partial U_{2}=\gamma \cup \partial_{\text {even }} U$. Let $\widehat{U}_{1}$ and $\widehat{U}_{2}$ be the respective pre-images of $U_{1}$ and $U_{2}$ in $\widehat{U}$. 
Since $K$ is disjoint from $U_{2}$, the covering $\widehat{U}_{2} \rightarrow U_{2}$ is trivial, and $\widehat{U}_{2}$ consists of two disjoint copies of the surface $U_{2}$ which are exchanged by $\sigma$.

The covering $\widehat{U}_{1} \rightarrow U_{1}$ is non-trivial above each component of $\partial_{\text {odd }} U$ and trivial above $\gamma$. Since the surface $U_{1}$ is planar, an Euler characteristic computation shows that $\widehat{U}_{1}$ has genus $\frac{1}{2} n_{\text {odd }}-1$ and has $n_{\text {odd }}+2$ boundary components.

Consider the Mayer-Vietoris exact sequence

$$
0 \rightarrow H_{1}(\widehat{\gamma} ; \mathbb{Z}) \rightarrow H_{1}\left(\widehat{U}_{1} ; \mathbb{Z}\right) \oplus H_{1}\left(\widehat{U}_{2} ; \mathbb{Z}\right) \rightarrow H_{1}(\widehat{U} ; \mathbb{Z}) \rightarrow 0
$$

where $\widehat{\gamma}$ denotes the pre-image of $\gamma$ in $\widehat{U}$. (To explain the 0 on the right, note that the map $H_{0}(\widehat{\gamma} ; \mathbb{Z}) \rightarrow H_{0}\left(\widehat{U}_{2} ; \mathbb{Z}\right)$ is injective. $)$

Lemma 30. Remembering that $V^{-}$denotes the $(-1)$-eigenspace of the action of $\sigma_{*}$ over a space $V$, the above exact sequence induces another exact sequence

$$
0 \rightarrow H_{1}(\widehat{\gamma} ; \mathbb{Z})^{-} \rightarrow H_{1}\left(\widehat{U}_{1} ; \mathbb{Z}\right)^{-} \oplus H_{1}\left(\widehat{U}_{2} ; \mathbb{Z}\right)^{-} \rightarrow H_{1}(\widehat{U} ; \mathbb{Z})^{-} \rightarrow 0
$$

Proof. The only point that requires some thought is the fact that the third homomorphism is surjective.

Given $u \in H_{1}(\widehat{U} ; \mathbb{Z})^{-}$, the first exact sequence provides $u_{1} \in H_{1}\left(\widehat{U}_{1} ; \mathbb{Z}\right)$ and $u_{2} \in H_{1}\left(\widehat{U}_{2} ; \mathbb{Z}\right)$ such that $u=u_{1}+u_{2}$ in $H_{1}(\widehat{U} ; \mathbb{Z})$. Since $\sigma_{*}(u)=-u$, we conclude that there exists $v \in H_{1}(\widehat{\gamma} ; \mathbb{Z})$ such that $\sigma_{*}\left(u_{1}\right)=-u_{1}+v$ in $H_{1}\left(\widehat{U}_{1} ; \mathbb{Z}\right)$ and $\sigma_{*}\left(u_{2}\right)=-u_{2}-v$ in $H_{1}\left(\widehat{U}_{2} ; \mathbb{Z}\right)$. Note that $v \in H_{1}(\widehat{\gamma} ; \mathbb{Z})$ is invariant under $\sigma_{*}$. Therefore, for the isomorphism $H_{1}(\widehat{\gamma} ; \mathbb{Z}) \cong$ $H_{1}(\gamma ; \mathbb{Z}) \oplus H_{1}(\gamma ; \mathbb{Z})$ coming from the fact that each of the two components of $\widehat{\gamma}$ is naturally identified to $\gamma, v=(w, w)$ for some $w \in H_{1}(\gamma ; \mathbb{Z})$. If we replace $u_{1}$ by $u_{1}^{\prime}=u_{1}-(w, 0)$ and $u_{2}$ by $u_{2}^{\prime}=u_{2}+(w, 0)$, we now have that $u=u_{1}^{\prime}+u_{2}^{\prime}$ with $\sigma_{*}\left(u_{1}^{\prime}\right)=-u_{1}^{\prime}$ and $\sigma_{*}\left(u_{2}^{\prime}\right)=-u_{2}^{\prime}$, as requested.

We now analyze the terms of the exact sequence of Lemma 30.

The space $H_{1}\left(\widehat{U}_{2} ; \mathbb{Z}\right)^{-}$is easy to understand, because $\widehat{U}_{2}$ is made up of two disjoint copies of $U_{2}$, which are exchanged by the covering involution $\sigma$. Therefore, $H_{1}\left(\widehat{U}_{2} ; \mathbb{Z}\right) \cong H_{1}\left(U_{2} ; \mathbb{Z}\right) \oplus$ $H_{1}\left(U_{2} ; \mathbb{Z}\right)$ and, for this isomorphism, $H_{1}\left(\widehat{U}_{2} ; \mathbb{Z}\right)^{-}$corresponds to $\left\{(\alpha,-\alpha) ; \alpha \in H_{1}\left(U_{2} ; \mathbb{Z}\right)\right\}$. This defines an isomorphism $H_{1}\left(\widehat{U}_{2} ; \mathbb{Z}\right)^{-} \cong H_{1}\left(U_{2} ; \mathbb{Z}\right)$, for which the intersection form of $H_{1}\left(\widehat{U}_{2} ; \mathbb{Z}\right)^{-}$corresponds to twice the intersection form of $H_{1}\left(U_{2} ; \mathbb{Z}\right)$.

Lemma 31. There exists a basis for $H_{1}\left(\widehat{U}_{2} ; \mathbb{Z}\right)^{-}$in which the intersection form is block diagonal with $h$ blocks $\left(\begin{array}{cc}0 & -2 \\ 2 & 0\end{array}\right)$ and $n_{\text {even }}$ blocks $(0)$.

In addition, we can arrange that the basis elements corresponding to the blocks (0) are the images of generators of $H(\widehat{\alpha} ; \mathbb{Z})^{-} \cong \mathbb{Z}$ as $\widehat{\alpha} \subset \widehat{U}_{2}$ ranges over all preimages of components $\alpha$ of $\partial_{\text {even }} U$, and that a generator of $H_{1}(\widehat{\gamma} ; \mathbb{Z})^{-} \cong \mathbb{Z}$ is sent to the sum of these elements.

Proof. The surface $U_{2}$ has genus $h$ and has $n_{\text {even }}+1$ boundary components, and $\gamma$ is one of these boundary components. We can therefore find a basis for $H_{1}\left(U_{2} ; \mathbb{Z}\right)$ in which the intersection form is block diagonal with $h$ blocks $\left(\begin{array}{cc}0 & -1 \\ 1 & 0\end{array}\right)$ and $n_{\text {even blocks }}(0)$. In addition, since $\partial U_{2}=\gamma \cup \partial_{\text {even }} U$, we can arrange that the basis elements corresponding to the blocks $(0)$ are the images of generators of $H_{1}(\alpha ; \mathbb{Z})$ as $\alpha$ ranges over all components of $\partial_{\text {even }} U$, while the image of a generator of $H_{1}(\gamma ; \mathbb{Z})$ is sent to the sum of these elements. 
The result then follows by considering the isomorphism $H_{1}\left(\widehat{U}_{2} ; \mathbb{Z}\right)^{-} \cong H_{1}\left(U_{2} ; \mathbb{Z}\right)$ mentioned above.

We now consider $H_{1}\left(\widehat{U}_{1} ; \mathbb{Z}\right)^{-}$.

Lemma 32. There exists a basis for $H_{1}\left(\widehat{U}_{1} ; \mathbb{Z}\right)^{-}$in which the intersection form is block diagonal with $\frac{1}{2} n_{\text {odd }}-1$ blocks $\left(\begin{array}{cc}0 & 4 \\ -4 & 0\end{array}\right)$ and with one block $(0)$. In addition, the block (0) corresponds to the image of the homomorphism $H_{1}(\widehat{\gamma} ; \mathbb{Z})^{-} \rightarrow H_{1}\left(\widehat{U}_{1} ; \mathbb{Z}\right)^{-}$induced by the inclusion map.

Proof. We will use an explicit description of the covering $\widehat{U}_{1} \rightarrow U_{1}$, with a specific basis for $H_{1}\left(\widehat{U}_{1} ; \mathbb{Z}\right)$.

Recall that this covering is classified by a cohomology class in $H^{1}\left(U_{1} ; \mathbb{Z}_{2}\right)$ that is dual to a family $K \subset U_{1}$ of $\frac{1}{2} n_{\text {odd }}$ disjoint arcs, with one boundary point in each component of $\partial_{\text {odd }} U$. Index the components of $\partial_{\text {odd }} U$ as $\alpha_{1}, \alpha_{2}, \ldots, \alpha_{n_{\text {odd }}}$ and the components of $K$ as $k_{1}, k_{3}, k_{5}$, $\ldots, k_{n_{\text {odd }}-1}$ in such a way that $k_{2 i-1}$ joins $\alpha_{2 i-1}$ to $\alpha_{2 i}$. Add to $K$ a family of disjoint arcs $k_{2}$, $k_{4}, \ldots, k_{n_{\text {odd }}-2}$, disjoint from the $k_{2 i-1}$, such that each $k_{2 i}$ joins $\alpha_{2 i}$ to $\alpha_{2 i+1}$. See Figure 3 ,

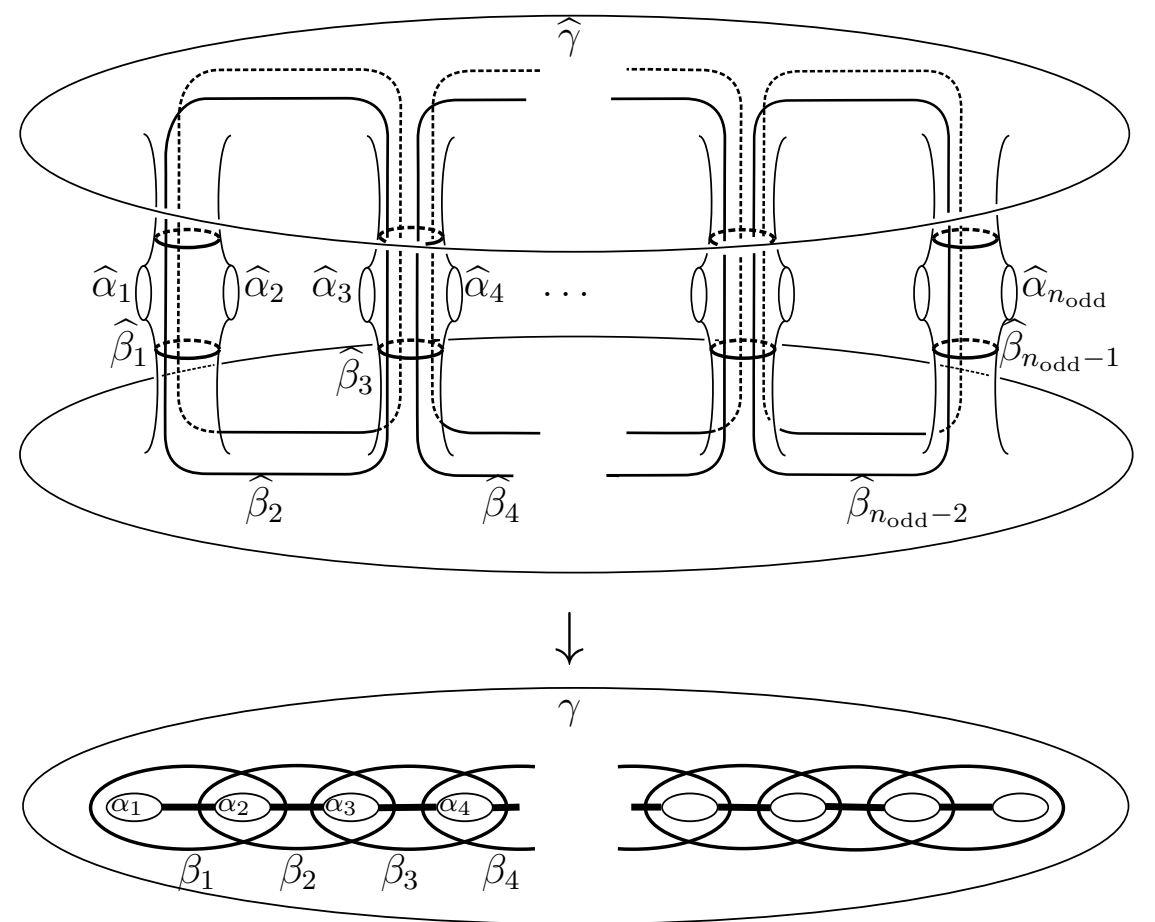

FigURE 3.

For $i=1,2, \ldots, n_{\text {odd }}-1$, consider a small regular neighborhood of $k_{i} \cup \alpha_{i} \cup \alpha_{i+1}$ in $U_{1}$ and let $\beta_{i}$ be the boundary component of this neighborhood which is neither $\alpha_{i}$ nor $\alpha_{i+1}$; endow $\beta_{i}$ by the corresponding boundary orientation. Orient each curve $\alpha_{i}$ by the boundary orientation of $\partial_{\text {odd }} U$.

The pre-image of each curve $\alpha_{i}$ is a single curve $\widehat{\alpha}_{i}$, which we orient by the orientation of $\alpha_{i}$. The pre-image of $\beta_{j}$ in $\widehat{U}_{1}$ consists of two disjoint curves. Arbitrarily choose one of 
these curves $\widehat{\beta}_{j}$ and orient it by the orientation of $\beta_{j}$. Then, the $\left[\widehat{\alpha}_{i}\right]$ and $\left[\widehat{\beta}_{j}\right]$ form a basis for $H_{1}\left(\widehat{U}_{1} ; \mathbb{Z}\right)$. See Figure 3 ,

Consider an element $u \in H_{1}\left(\widehat{U}_{1} ; \mathbb{Z}\right)$, uniquely expressed in this basis as

$$
u=\sum_{i=1}^{n_{\text {odd }}} a_{i}\left[\widehat{\alpha}_{i}\right]+\sum_{j=1}^{n_{\text {odd }}-1} b_{j}\left[\widehat{\beta}_{j}\right]
$$

with all $a_{i}, b_{j} \in \mathbb{Z}$. By construction of the curves $\widehat{\alpha}_{i}$ and $\widehat{\beta}_{j}$,

$$
\sigma_{*}\left(\left[\widehat{\alpha}_{i}\right]\right)=\left[\widehat{\alpha}_{i}\right] \text { and } \sigma_{*}\left(\left[\widehat{\beta}_{j}\right]\right)=-\left[\widehat{\beta}_{j}\right]-\left[\widehat{\alpha}_{j}\right]-\left[\widehat{\alpha}_{j+1}\right] .
$$

If $u$ belongs to $H_{1}\left(\widehat{U}_{1} ; \mathbb{Z}\right)^{-}$, namely if $\sigma_{*}(u)=-u$, it follows from these observations and from the consideration of the coefficients of each $\left[\widehat{\alpha}_{i}\right]$ that we necessarily have

$$
\begin{aligned}
b_{1} & =2 a_{1}, \\
b_{i}+b_{i-1} & =2 a_{i} \text { for every } i \text { with } 2 \leqslant i \leqslant n_{\text {odd }}-1, \\
\text { and } b_{n_{\text {odd }}-1} & =2 a_{n_{\text {odd }}} .
\end{aligned}
$$

In particular, the coefficients $b_{j}$ are all even, and

$$
u=\frac{u-\sigma_{*}(u)}{2}=\sum_{j=1}^{n_{\text {odd }}-1} \frac{b_{j}}{2}\left(\left[\widehat{\beta}_{j}\right]-\sigma_{*}\left(\left[\widehat{\beta}_{j}\right]\right)\right) .
$$

Therefore, the elements $\left[\widehat{\beta}_{j}\right]-\sigma_{*}\left(\left[\widehat{\beta}_{j}\right]\right)$ generate $H_{1}\left(\widehat{U}_{1} ; \mathbb{Z}\right)^{-}$. Since these elements $\left[\widehat{\beta}_{j}\right]-$ $\sigma_{*}\left(\left[\widehat{\beta}_{j}\right]\right)=2\left[\widehat{\beta}_{j}\right]+\left[\widehat{\alpha}_{j}\right]+\left[\widehat{\alpha}_{j+1}\right]$ are linearly independent, they form a basis for $H_{1}\left(\widehat{U}_{1} ; \mathbb{Z}\right)^{-}$.

Note that $\left[\widehat{\beta}_{j}\right] \cdot\left[\widehat{\beta}_{j^{\prime}}\right]=0$ if $\left|j-j^{\prime}\right|>1$, and $\left[\widehat{\beta}_{j}\right] \cdot\left[\widehat{\beta}_{j+1}\right]=\varepsilon_{j}= \pm 1$, where the sign depend on which lift of $\beta_{j}$ we chose for $\widehat{\beta}_{j}$. Also,

$$
\sigma_{*}\left(\left[\widehat{\beta}_{j}\right]\right) \cdot\left[\widehat{\beta}_{j^{\prime}}\right]=\left[\widehat{\beta}_{j}\right] \cdot \sigma_{*}\left(\left[\widehat{\beta}_{j^{\prime}}\right]\right)=-\sigma_{*}\left(\left[\widehat{\beta}_{j}\right]\right) \cdot \sigma_{*}\left(\left[\widehat{\beta}_{j^{\prime}}\right]\right)=-\left[\widehat{\beta}_{j}\right] \cdot\left[\widehat{\beta}_{j^{\prime}}\right] .
$$

It follows that, in the basis of $H_{1}\left(\widehat{U}_{1} ; \mathbb{Z}\right)^{-}$formed by the $\left[\widehat{\beta}_{j}\right]-\sigma_{*}\left(\left[\widehat{\beta}_{j}\right]\right)$, the intersection form has matrix

$$
\left(\begin{array}{ccccccc}
0 & 4 \varepsilon_{1} & 0 & 0 & \ldots & 0 & 0 \\
-4 \varepsilon_{1} & 0 & 4 \varepsilon_{2} & 0 & \ldots & 0 & 0 \\
0 & -4 \varepsilon_{2} & 0 & 4 \varepsilon_{3} & \ldots & 0 & 0 \\
0 & 0 & -4 \varepsilon_{3} & 0 & \ldots & 0 & 0 \\
\ldots & \ldots & \ldots & \ldots & \ldots & \ldots & \ldots \\
0 & 0 & 0 & 0 & \ldots & 0 & 4 \varepsilon_{n_{\text {odd }}-2} \\
0 & 0 & 0 & 0 & \ldots & -4 \varepsilon_{n_{\text {odd }}-2} & 0
\end{array}\right)
$$

By block diagonalizing this matrix, a final modification of the basis provides a new basis for $H_{1}\left(\widehat{U}_{1} ; \mathbb{Z}\right)^{-}$in which the intersection form is block diagonal with $\frac{1}{2} n_{\text {odd }}-1$ blocks $\left(\begin{array}{cc}0 & 4 \\ -4 & 0\end{array}\right)$ and with one block $(0)$.

There remains to show that the block (0) corresponds to the image of $H_{1}(\widehat{\gamma} ; \mathbb{Z})^{-}$. This could be seen by explicitly analyzing the block diagonalization process of the above matrix. However, it is easier to note that $H_{1}(\widehat{\gamma} ; \mathbb{Z})^{-} \cong \mathbb{Z}$ is generated by $\left[\widehat{\gamma}_{1}\right]-\left[\widehat{\gamma}_{2}\right]$, where $\widehat{\gamma}_{1}$ and $\widehat{\gamma}_{2}$ are the two components of the pre-image $\widehat{\gamma}$ of $\gamma$ and are oriented by the boundary orientation of $\partial \widehat{U}_{1}$. Then, $\left[\widehat{\gamma}_{1}\right]-\left[\widehat{\gamma}_{2}\right]$ is in the kernel of the intersection form of $H_{1}\left(\widehat{U}_{1} ; \mathbb{Z}\right)^{-}$, since $\widehat{\gamma}_{1}$ and 
$\widehat{\gamma}_{2}$ are in the boundary of $\widehat{U}_{1}$, and generate this kernel since it is isomorphic to $\mathbb{Z}$ and since $\left[\widehat{\gamma}_{1}\right]-\left[\widehat{\gamma}_{2}\right]$ is indivisible in $H_{1}\left(\widehat{U}_{1} ; \mathbb{Z}\right)$.

We now only need to combine the computations of Lemmas 30, 31 and 32 to obtain a basis of $H_{1}(\widehat{U} ; \mathbb{Z})^{-}$in which the intersection form is block diagonal with $h$ blocks $\left(\begin{array}{cc}0 & -2 \\ 2 & 0\end{array}\right)$, $\frac{1}{2} n_{\text {odd }}-1$ blocks $\left(\begin{array}{cc}0 & -4 \\ 4 & 0\end{array}\right)$, and $n_{\text {even }}$ blocks $(0)$.

Applying Lemmas 27 and 28 to connect this to the Thurston intersection form on the edge weight space $\mathcal{W}(\tau ; \mathbb{Z})$, we conclude that $\mathcal{W}(\tau ; \mathbb{Z})$ admits a basis in which the intersection form is block diagonal with $h$ blocks $\left(\begin{array}{cc}0 & -1 \\ 1 & 0\end{array}\right), \frac{1}{2} n_{\text {odd }}-1$ blocks $\left(\begin{array}{cc}0 & -2 \\ 2 & 0\end{array}\right)$, and $n_{\text {even }}$ blocks $(0)$. In addition, by the second half of Lemma 31 and using Lemma 29, the generators corresponding to the blocks $(0)$ can be assumed to correspond to the elements of $\mathcal{W}(\tau ; \mathbb{Z})$ associated to the components of $\partial_{\text {even }} U$.

This proves Theorem 26, under our assumption that $n_{\text {odd }}>0$.

We now consider the case where $n_{\text {odd }}=0$, namely where $\partial_{\text {odd }} U=\varnothing$, and where the train-track $\tau$ is non-orientable. This second property is equivalent to the property that the covering $\widehat{U} \rightarrow U$ is non-trivial. We can then realize the cohomology class of $H^{1}\left(U ; \mathbb{Z}_{2}\right)$ classifying the covering $\widehat{U} \rightarrow U$ as the Poincaré dual of a non-separating simple closed curve $K$. Let $U_{1} \subset U$ be a surface of genus 1 containing $K$ and bounded by a simple closed curve $\gamma$, and let $U_{2}$ be the closure of $U-U_{1}$. As before, let $\widehat{U}_{1}, \widehat{U}_{2}, \widehat{\gamma}$ denote the respective pre-images of $U_{1}, U_{2}, \gamma$ in $\widehat{U}$.

The computation of Lemma 31 applies to this case as well, and provides a basis for $H_{1}\left(\widehat{U}_{2} ; \mathbb{Z}\right)^{-}$in which the intersection form is block diagonal with $h$ blocks $\left(\begin{array}{cc}0 & -2 \\ 2 & 0\end{array}\right)$ and $n_{\text {even }}$ blocks $(0)$.

The surface $\widehat{U}_{1}$ is a twice-punctured torus. A simple analysis of the covering $\widehat{U}_{1} \rightarrow U_{1}$ shows that $H_{1}\left(\widehat{U}_{1} ; \mathbb{Z}\right)^{-} \cong \mathbb{Z}$ is equal to the image of $H_{1}(\widehat{\gamma} ; \mathbb{Z})^{-}$. The intersection form of $H_{1}\left(\widehat{U}_{1} ; \mathbb{Z}\right)^{-}$is then 0 .

Again, combining these computations with the exact sequence

$$
0 \rightarrow H_{1}(\widehat{\gamma} ; \mathbb{Z})^{-} \rightarrow H_{1}\left(\widehat{U}_{1} ; \mathbb{Z}\right)^{-} \oplus H_{1}\left(\widehat{U}_{2} ; \mathbb{Z}\right)^{-} \rightarrow H_{1}(\widehat{U} ; \mathbb{Z})^{-} \rightarrow 0 .
$$

provides in this case a basis for $H_{1}(\widehat{U} ; \mathbb{Z})^{-}$in which the intersection form is block diagonal with $h$ blocks $\left(\begin{array}{cc}0 & -2 \\ 2 & 0\end{array}\right)$ and $n_{\text {even }}$ blocks (0). Using Lemmas 27 and 28, this provides the result promised in Theorem 26]in this case as well. The fact that the generators corresponding to the blocks $(0)$ can be chosen to be the elements associated to the components of $\partial_{\text {even }} U$ is a byproduct of the proof as in the previous case.

Finally, we need to consider the case where $n_{\text {odd }}=0$ and the train-track $\tau$ is orientable. Then the covering $\widehat{U} \rightarrow U$ is trivial, so that $H_{1}(\widehat{U} ; \mathbb{Z})^{-} \cong H_{1}(U ; \mathbb{Z})$ in such a way that the intersection form of $H_{1}(\widehat{U} ; \mathbb{Z})^{-}$corresponds to twice the intersection form of $H_{1}(U ; \mathbb{Z})$. By Lemma 28, the last case of Theorem 26] immediately follows. 


\section{REFERENCES}

[AbF $\left.{ }_{1}\right]$ Nel Abdiel, Charles Frohman, Frobenius algebras derived from the Kauffman bracket skein algebra, preprint, 2014, arXiv: 1412.4144.

$\left[\mathrm{AbF}_{2}\right]$ Nel Abdiel, Charles Frohman, The localized skein algebra is Frobenius, preprint, 2015, arXiv: 1501.02631.

[Ba] John W. Barrett, Skein spaces and spin structures, Math. Proc. Cambridge Philos. Soc. 126 (1999), $267-275$.

[BHMV] Christian Blanchet, Nathan Habegger, Gregor Masbaum, Pierre Vogel, Topological quantum field theories derived from the Kauffman bracket, Topology 34 (1995), 883-927.

[Bo] Francis Bonahon, Shearing hyperbolic surfaces, bending pleated surfaces and Thurston's symplectic form, Ann. Fac. Sci. Toulouse Math. 5 (1996), 233-297.

[BoL] Francis Bonahon, Xiaobo Liu, Representations of the quantum Teichmüller space and invariants of surface diffeomorphisms, Geom. Topol. 11 (2007), 889-938.

$\left[\mathrm{BoW}_{1}\right]$ Francis Bonahon, Helen Wong, Quantum traces for representations of surface groups in $\mathrm{SL}_{2}(\mathbb{C})$, Geometry \& Topology 15 (2011), 1569-1615.

$\left[\mathrm{BoW}_{2}\right]$ Francis Bonahon, Helen Wong, Kauffman brackets, character varieties and triangulations of surfaces, in Topology and Geometry in Dimension Three: Triangulations, Invariants, and Geometric Structures (W. Li, L. Bartolini, J. Johnson, F. Luo, R. Myers, J. H. Rubinstein eds.), Contemporary Mathematics 560, American Math. Society, 2011.

$\left[\mathrm{BoW}_{3}\right]$ Francis Bonahon, Helen Wong, Representations of the Kauffman bracket skein algebra I: invariants and miraculous cancellations, Invent. Math. (2015), published electronically, DOI 10.1007/s00222015-0611-y.

$\left[\mathrm{BoW}_{4}\right]$ Francis Bonahon, Helen Wong, Representations of the Kauffman bracket skein algebra III: closed surfaces and naturality, submitted for publication, arXiv:1505.01522.

$\left[\mathrm{BoW}_{5}\right]$ Francis Bonahon, Helen Wong, The Witten-Reshetikhin-Turaev representation of the Kauffman bracket skein algebra, Proc. Amer. Math. Soc. (2015), published electronically, DOI $10.1090 / \mathrm{proc} / 12927$.

$\left[\mathrm{BoW}_{6}\right]$ Francis Bonahon, Helen Wong, Representations of the Kauffman bracket skein algebra IV: naturality for punctured surfaces, in preparation.

$\left[\mathrm{Bu}_{1}\right] \quad$ Doug Bullock, Estimating a skein module with $\mathrm{SL}_{2}(\mathbb{C})$ characters, Proc. Amer. Math. Soc. 125 (1997), 1835-1839.

$\left[\mathrm{Bu}_{2}\right] \quad$ Doug Bullock, Rings of $\mathrm{SL}_{2}(\mathbb{C})$-characters and the Kauffman bracket skein module, Comment. Math. Helv. 72 (1997), 521-542.

[BFK 1 Doug Bullock, Charles Frohman, Joanna Kania-Bartoszyńska, Understanding the Kauffman beacket skein module, J. Knot Theory Ramifications 8 (1999), 265-277.

$\left[\mathrm{BFK}_{2}\right]$ Doug Bullock, Charles Frohman, Joanna Kania-Bartoszyńska, The Kauffman bracket skein as an algebra of observables, Proc. Amer. Math. Soc. 130 (2002), 2479-2485.

[BuP] Doug Bullock, Józef H. Przytycki, Multiplicative structure of Kauffman bracket skein module quantizations, Proc. Amer. Math. Soc. 128 (2000), 923-931.

$\left[\mathrm{ChF}_{1}\right]$ Leonid O. Chekhov, Vladimir V. Fock, Quantum Teichmüller spaces, Theor.Math.Phys. 120 (1999) $1245-1259$.

$\left[\mathrm{ChF}_{2}\right]$ Leonid O. Chekhov, Vladimir V. Fock, Observables in 3D gravity and geodesic algebras, in: Quantum groups and integrable systems (Prague, 2000), Czechoslovak J. Phys. 50 (2000), 1201-1208.

[Fo] Vladimir V. Fock, Dual Teichmüller spaces, unpublished preprint, 1997, arXiv:Math/dg-ga/9702018 .

[HaP] Miloslav Havlíček, Severin Pošta, On the classification of irreducible finite-dimensional representations of $\mathrm{U}_{q}^{\prime}\left(\mathrm{SO}_{3}\right)$ algebra, J. Math. Physics 42 (2001), 472-500.

[Ka] Rinat Kashaev, Quantization of Teichmüller spaces and the quantum dilogarithm, Lett. Math. Phys. 43 (1998), 105-115.

[Lê] Thang T. Q. Lê, On Kauffman bracket skein modules at root of unity, Alg. Geom. Topol. 15 (2015), $1093-1117$.

[Liu] Xiaobo Liu, The quantum Teichmüller space as a non-commutative algebraic object, J. Knot Theory Ramifications 18 (2009), 705-726. 
[MFK] David Mumford, John Fogarty, Frances Kirwan, Geometric invariant theory. Third edition, Ergebnisse der Mathematik und ihrer Grenzgebiete 34, Springer-Verlag, 1994.

$[\mathrm{PeH}] \quad$ Robert C. Penner, John L. Harer, Combinatorics of train tracks, Annals of Mathematics Studies vol. 125, Princeton University Press, Princeton, 1992.

$[\mathrm{PrS}] \quad$ Jozef H. Przytycki, Adam S. Sikora, On skein algebras and $\mathrm{SL}_{2}(\mathbb{C})$-character varieties, Topology 39 (2000), 115-148.

[ReT] Nicolai Y. Reshetikhin and Vladimir G. Turaev, Invariants of 3-manifolds via link polynomials and quantum groups, Invent. Math. 103 (1991), 547-597.

[Ta] Nurdin Takenov, Representations of the Kauffman skein algebra of small surfaces, preprint, 2015, arXiv: 1504.04573.

[Th] William P. Thurston, The geometry and topology of 3-manifolds, Princeton lecture notes, 1978-1981

$\left[\mathrm{Tu}_{1}\right] \quad$ Vladimir Turaev, Skein quantization of Poisson algebras of loops on surfaces, Ann. Sci. École Norm. Sup. 24 (1991), 635-704.

$\left[\mathrm{Tu}_{2}\right] \quad$ Vladimir G. Turaev. Quantum invariants of knots and 3-manifolds, de Gruyter Studies in Mathematics Vol. 18, Walter de Gruyter \& Co., Berlin, 1994.

[Wit] Edward Witten, Quantum field theory and the Jones polynomial, Commun. Math. Phys. 121 (1989), $351-399$.

Department of Mathematics, University of Southern California, Los Angeles CA 900892532 , U.S.A.

E-mail address: fbonahon@math.usc.edu

Department of Mathematics, Carleton College, Northfield MN 55057, U.S.A.

E-mail address: hwong@carleton.edu 